\title{
Entwurf konvergenzoptimaler weich strukturvariabler Regelungen für lineare Systeme mit Stellgrößenbeschränkung
}

\author{
Design of convergence rate optimal soft variable structure controls for linear systems with \\ input saturation
}

Zusammenfassung: In diesem Beitrag wird eine einfache nichtlineare Zustandsrückführung für lineare Strecken mit Stellgrößenbeschränkung vorgestellt. Ziel ist es, schnelle Ausregelvorgänge durch die Verwendung immer schnellerer Regler bei gleichzeitiger maximaler Ausnutzung des Stellgrößenbereichs zu erzielen. Dies wird durch einen einfachen parameterabhängigen Zweipunktregler erreicht. Die Stabilitätsbedingungen und Entwurfsmethodik werden vorgestellt. Komparativ zu anderen nichtlinearen Regelungen erfolgt schließlich die Analyse dieser Konvergenzrate, die eine untere Grenze der tatsächlichen Konvergenzrate des Systems innerhalb des Einzugsgebietes der Ruhelage darstellt.

Schlüsselwörter: Konvergenzrate, Stellgrößenbeschränkung, weich strukturvariable Regelung mittels impliziter Ljapunov Funktionen.

Abstract: This article presents a simple nonlinear state feedback for linear systems with input saturation. The goal is to obtain fast control processes by using everaccelerating controls during regulation cycles and maximal exploitation of the actuating variable range. This is achieved by a simple parameter dependent bang-bang control. The stability conditions and design method are presented. In comparison with other nonlinear controls, the paper shows finally the analysis of this convergence rate, which is a lower bound of the overall convergence

\footnotetext{
*Korrespondenzautor: Andreea Röthig, Institut für Automatisierungstechnik und Mechatronik, Technische Universität Darmstadt, Landgraf-Georg-Str. 4, 64283 Darmstadt, E-Mail: aroethig@rtr.tu-darmstadt.de Jürgen Adamy: Institut für Automatisierungstechnik und Mechatronik, Technische Universität Darmstadt, Landgraf-GeorgStr. 4, 64283 Darmstadt
}

rate of the system for all trajectories inside the stability region.

Keywords: Convergence rate, input saturation, soft variable structure controls with implicit Lyapunov functions.

DOI 10.1515/auto-2014-1080

Eingang 8. Januar 2014; angenommen 1. Oktober 2014

\section{Einleitung}

Jedes reale Stellglied ist aus physikalischen Gründen mit einer Amplitudenbeschränkung behaftet. Im regelungstechnischen Bereich sind Systeme mit Stellgrößenbeschränkungen seit längerer Zeit Gegenstand vieler Untersuchungen, vgl. hierzu [5] und die darin enthaltenen Verweise. Der Entwurf verschiedener linearer und nichtlinearer Regelgesetze, sowie die Stabilitätsanalyse der Gesamtsysteme werden dabei grundsätzlich auf zwei Weisen angegangen: entweder durch die Vernachlässigung der Sättigungsglieder beim Regelungsentwurf und die nachträgliche Einführung von speziellen Strukturen, die sogenannten Anti-Windup-Strukturen [19], welche die Sättigungseffekte vermindern, oder durch die systematische Behandlung der Sättigungsglieder sowohl beim Reglerentwurf als auch im Rahmen der Stabilitätsanalyse, vgl. hierzu [8]. Die typischen Anforderungen an diese nichtlinearen Regelkreise sind dabei große Stabilitätsgebiete und schnelle Ausregelvorgänge, welche jedoch im Allgemeinen in Konflikt stehen.

Als Gütemaß für die Schnelligkeit des Ausregelvorgangs wird oft die Konvergenzrate verwendet, vgl. hier$\mathrm{zu}[8,11,12,14,15,20]$, d.h. die Abklingrate einer Exponentialfunktion, welche als kleinste obere Schranke der Zustandsnorm fungiert, weil je höher die Konvergenzrate 
ist, desto kürzer ist im Allgemeinen die Ausregelzeit. Dabei wird der Zusammenhang zwischen der Konvergenzrate eines exponentiell stabilen Systems und der Konvergenzrate einer Ljapunov-Funktion entlang der Trajektorien des Systems verwendet, welche die Stabilität des Systems gewährleistet. Oft werden in diesem Kontext quadratische Ljapunov-Funktionen angenommen, vgl. [8, 10, 12, 15], mit dem Vorteil, dass das entsprechende Regelungsziel, die Maximierung der Konvergenzrate der Ljapunov-Funktion, mit Hilfe von linearen Matrixungleichungen (LMI), vgl. [6], formuliert werden kann.

Im Gegensatz zum Fall linearer Systeme ist die Konvergenzrate nichtlinearer Systeme abhängig vom betrachteten Ljapunov-Gebiet, wobei im Allgemeinen gilt: je kleiner der Abstand zur Ruhelage, desto höher die Konvergenzrate. Wie in diesem Beitrag gezeigt wird, kann diese Variation gezielt durch den Reglerentwurf beeinflusst werden. Es handelt sich hier um eine sättigende strukturvariable Regelung mit einem parameterabhängigen Zustandsregelgesetz, wobei die Reglerverstärkung kontinuierlich an den Abstand zur Ruhelage angepasst wird. Diese gehört zu der Klasse weich strukturvariabler Regler, vgl. [3] und die darin enthaltenen Verweise.

Im Fall weich strukturvariabler Regelungen ohne Gleitzustand wurden für lineare Strecken mit Stellgrößenbeschränkung verschiedene parameterabhängige Regelgesetze vorgeschlagen, die mit der Verkleinerung des Abstandes zur Ruhelage immer größere Auswirkung auf die Strecke haben und dabei im sättigenden Bereich arbeiten können. Sie weisen unterschiedliche Selektionsstrategien auf, welche das Einzugsgebiet der Ruhelage in infinitesimal dicht ineinander verschachtelte Teilgebiete trennen, vgl. [7, 9, 11, 15]. Ein gemeinsames Entwurfsziel ist dabei die Maximierung der Konvergenzrate einer LjapunovFunktion entlang der Trajektorien der mit dem schwächsten Regler geregelten Strecke.

Will man die Konvergenzrate in jedem Teilgebiet und relativ zu allen möglichen beschränkten Regelgesetzen maximieren, so ergibt sich ein Zweipunktregler, der nur von der gewählten parameterabhängigen LjapunovMatrix abhängig ist. Die Selektionsstrategie und mithin die Wahl der parameterabhängigen Ljapunov-Matrix hat folglich einen direkten Einfluß auf die Konvergenzrate des Gesamtsystems.

Der Beitrag gliedert sich wie folgt. In Abschnitt 2 wird die Konvergenzrate eines nichtlinearen Systems im Zusammenhang mit der Konvergenzrate einer LjapunovFunktion vorgestellt. In Abschnitt 3 wird die sättigende lineare Regelung aus [8] wiederaufgenommen und in Abschnitt 4 die konvergenzoptimale weich strukturvariable Regelung mittels impliziter Ljapunov-Funktionen vorge- stellt. Dabei werden in Abschnitt 4.1 die Stabilitätsbedingungen und Entwurfsmethodik vorgestellt und in Abschnitt 4.2 die Konvergenzraten verschiedener strukturvariabler Regelungen komparativ analysiert. In Abschnitt 4.3 veranschaulicht das Beispiel eines Systems zweiter Ordnung die Variation der Konvergenzraten verschiedener strukturvariabler Regelungen. In Abschnitt 5 werden die Ergebnisse zusammengefasst und es wird ein Ausblick gegeben.

\subsection{Vorbemerkungen}

Folgende Notationen werden verwendet:

$\mathbb{P}^{n \times n} \quad n \times n$ positiv definite Matrizen

$\mathbb{N}^{n \times n} \quad n \times n$ positiv semidefinite Matrizen

$\mathbb{H}^{n \times n} \quad n \times n$ hermitesche Matrizen

$\mathbf{0}_{m, n} \quad m \times n$ Nullmatrix

$I_{n} \quad n \times n$ Einheitsmatrix

$\boldsymbol{A}>\boldsymbol{B} \quad \Leftrightarrow \boldsymbol{A}-\boldsymbol{B} \in \mathbb{P}^{n \times n}$

$\boldsymbol{A} \geq \boldsymbol{B} \quad \Leftrightarrow \boldsymbol{A}-\boldsymbol{B} \in \mathbb{N}^{n \times n}$

$\operatorname{Spec}(\boldsymbol{A}) \quad$ Spektrum einer Matrix $\boldsymbol{A} \in \mathbb{R}^{n \times n}$

$\lambda(A) \in \mathbb{C} \quad$ Eigenwert einer Matrix $A \in \mathbb{R}^{n \times n}$

$\operatorname{Re} \lambda(A) \quad$ Realteil des Eigenwertes $\lambda \in \operatorname{Spec}(A)$

$\lambda_{\text {max }}(A) \in \mathbb{R}$ größter Eigenwert einer Matrix $A$

$\lambda_{\text {min }}(A) \in \mathbb{R}$ kleinster Eigenwert einer Matrix $A$

$a_{(i, j)} \quad$ Element $(i, j)$ einer Matrix $\boldsymbol{A}$

$\boldsymbol{x}^{\boldsymbol{T}} \in \mathbb{R}^{n} \quad$ Zeilenvektor

$\begin{array}{lll}x_{\lambda} & \text { zum Eigenwert } \lambda \text { gehörender Rechtseigen }\end{array}$ vektor

$\partial G \quad$ Rand eines abgeschlossenen Gebiets $G$

$\boldsymbol{P}_{v} \quad$ vom Parameter $v$ abhängige Matrix $\boldsymbol{P}(v) \epsilon$ $\mathbb{R}^{n \times n}$

$A \mid \mathcal{A} \quad$ Schur-Komplement von $A$ bzgl. $\quad \mathcal{A}=$ $\left[\begin{array}{ll}\boldsymbol{A} & \boldsymbol{B} \\ \boldsymbol{C} & \boldsymbol{D}\end{array}\right]$ definiert als $\boldsymbol{A} \mid \mathcal{A}=\boldsymbol{D}-\boldsymbol{C A}^{-1} \boldsymbol{B}$, $A$ nichtsingulär

$\|x\| \quad$ Euklidische Norm eines Vektors $\boldsymbol{x} \in \mathbb{R}^{n}$

\section{Konvergenzrate eines exponentiell stabilen dynamischen Systems}

Die Konvergenzrate eines exponentiell stabilen nichtlinearen Systems kann im Zusammenhang mit der Konvergenzrate einer Ljapunov-Funktion entlang der Trajektorien des Systems untersucht werden, welche die Stabilität des Gesamtsystems gewährleistet. So wie im Weiteren gezeigt wird, bietet die Konvergenzrate der Ljapunov-Funktion, 
die entlang der Trajektorien des Systems variabel ist, eine untere Grenze der ebenfalls variablen Konvergenzrate des Gesamtsystems.

Definition 1 (Konvergenzrate eines exponentiell stabilen Systems). Der maximale Abklingfaktor $\alpha>0$ eines exponentiell stabilen Systems $\dot{\boldsymbol{x}}=\boldsymbol{f}(\boldsymbol{x}), \boldsymbol{x} \in \mathbb{R}^{n}$, wofür $\exists \gamma>0$, sodass $\|x\| \leq \gamma\left\|x_{0}\right\| e^{-\alpha t}, \forall t>0$ gilt, wird Konvergenzrate des Systems genannt.

Für stabile lineare Systeme $\dot{\boldsymbol{x}}=\boldsymbol{A}_{\mathrm{g}} \boldsymbol{x}, \boldsymbol{x} \in \mathbb{R}^{n}$ kann eine untere Grenze der Konvergenzrate in der Form $\underline{\alpha}_{V}=$ $0.5 \lambda_{\text {min }}(\mathbf{Q}) / \lambda_{\text {max }}(\mathbf{P})$, mit $\boldsymbol{P}, \boldsymbol{Q}>\mathbf{0}$ mittels einer quadratischen Ljapunov-Funktion $V(\boldsymbol{x})=\boldsymbol{x}^{\top} \boldsymbol{P} \boldsymbol{x}$ angegeben werden [18], die für eine beliebige Matrix $\boldsymbol{Q} \succ \boldsymbol{0}$ und $\boldsymbol{A}_{\mathrm{g}}^{\top} \boldsymbol{P}+\boldsymbol{P} \boldsymbol{A}_{\mathrm{g}}=$ $-\mathbf{Q}$, die Ungleichung

$$
\dot{V}(\boldsymbol{x}) \leq-2 \underline{\alpha}_{V} V(\boldsymbol{x}), \forall \boldsymbol{x} \in \mathbb{R}^{n},
$$

und folglich $V(\boldsymbol{x}) \leq V\left(\boldsymbol{x}_{0}\right) e^{-2 \underline{\alpha}_{V} t}, \forall \boldsymbol{x} \in \mathbb{R}^{n}$ erfüllt. Zusammen mit

$$
\lambda_{\min }(\boldsymbol{P})\|\boldsymbol{x}\|^{2}=\boldsymbol{x}^{\top} \lambda_{\min }(\boldsymbol{P}) \boldsymbol{I} \boldsymbol{x} \leq \boldsymbol{x}^{\top} \boldsymbol{P} \boldsymbol{x}, \forall \boldsymbol{x} \neq \mathbf{0},
$$

folgt, dass $\|x\| \leq \sqrt{V\left(\boldsymbol{x}_{0}\right) / \lambda_{\min }(\boldsymbol{P})} e^{-\underline{\alpha}_{V} t}, \forall \boldsymbol{x} \in \mathbb{R}^{n}$, d.h. der Abklingfaktor $\alpha$ ist mindestens $\underline{\alpha}_{V}$. Diese untere Grenze kann mit Hilfe des konvexen Optimierungsproblems

$$
\begin{aligned}
& \max _{\boldsymbol{P}} \underline{\alpha}_{V}, \text { sodass } \\
& \boldsymbol{P} \succ \mathbf{0}, \boldsymbol{A}_{\mathrm{g}}^{\top} \boldsymbol{P}+\boldsymbol{P} \boldsymbol{A}_{\mathrm{g}}+2 \underline{\alpha}_{V} \boldsymbol{P} \preceq \mathbf{0} .
\end{aligned}
$$

maximiert werden. Die Lösung $\boldsymbol{P}^{*}$ liefert die maximale Untergrenze $\underline{\alpha}_{V}^{*}$ der Konvergenzrate und entspricht bei linearen Systemen dem Realteil des dominanten Eigenwertes der Matrix $\boldsymbol{A}_{\mathrm{g}}$, d.h.

$$
\alpha=\underline{\alpha}_{V}^{*}=-\max _{i}\left\{\operatorname{Re} \lambda_{i}\left(A_{\mathrm{g}}\right)\right\} .
$$

Im Allgemeinen kann man auch für nichtlineare Systeme eine untere Grenze der Konvergenzrate mittels Ljapunov-Funktionen und der dazugehörigen kontraktiv invarianten Gebiete angeben. Verwendet wird folgende Definition eines kontraktiv invarianten Gebietes eines nichtlinearen Systems:

Definition 2. Ein abgeschlossenes Gebiet

$$
G(V, c)=\left\{x \in \mathbb{R}^{n} \mid V(x) \leq c\right\}
$$

heißt kontraktiv invariant für ein System $\dot{\boldsymbol{x}}=\boldsymbol{f}(\boldsymbol{x})$ mit der Ruhelage $\boldsymbol{x}_{\mathrm{R}}=\mathbf{0}$, wenn für eine gegebene Funktion $V(\boldsymbol{x})$, mit $V(\boldsymbol{x})>0, \forall \boldsymbol{x} \in G(V, c) \backslash\{\mathbf{0}\}$, und $V(\mathbf{0})=0, \dot{V}(\boldsymbol{x})<$ $0, \forall \boldsymbol{x} \in G(V, c) \backslash\{\boldsymbol{0}\}$ gilt. Somit ist $V(\boldsymbol{x})$ eine LjapunovFunktion des Systems und das Gebiet $G(V, c)$ ist Teil des Einzugsgebietes der Ruhelage $\boldsymbol{x}_{\mathrm{R}}=\mathbf{0}$.
Bei quadratischen Ljapunov-Funktionen ist dieses Gebiet ellipsoidal, d.h. $\mathcal{E}(\boldsymbol{P}, c):=G(V, c)=\left\{\boldsymbol{x} \in \mathbb{R}^{n} \mid \boldsymbol{x}^{\top} \boldsymbol{P} \boldsymbol{x} \leq c\right\}$.

Lemma 1. Für das exponentiell stabile nichtlineare System $\dot{\boldsymbol{x}}=\boldsymbol{f}(\boldsymbol{x}), \boldsymbol{x} \in \mathbb{R}^{n}$ mit dem kontraktiv invarianten Gebiet $G(V, c)$ stellt die größte untere Schranke der Konvergenzrate der Ljapunov-Funktion $V(\boldsymbol{x})=\boldsymbol{x}^{\top} \boldsymbol{P} \boldsymbol{x}$,

$$
\underline{\alpha}_{V}(c)=\frac{1}{2} \cdot \inf _{x \in G(V, c) \backslash\{0\}}\left(-\frac{\dot{V}(\boldsymbol{x})}{V(\boldsymbol{x})}\right)
$$

eine Untergrenze der Konvergenzrate $\alpha$ des Systems innerhalb des Gebietes $G(V, c) \backslash\{\mathbf{0}\}$ dar.

Beweis. Für jeden Zustand $\boldsymbol{x} \in G(V, c) \backslash\{\mathbf{0}\}$ existiert für das exponentiell stabile nichtlineare System $\dot{\boldsymbol{x}}=\boldsymbol{f}(\boldsymbol{x})$ ein $\alpha_{x}>0$, sodass $\dot{V}(\boldsymbol{x}) / V(\boldsymbol{x}) \leq-2 \alpha_{x}$, d.h. $\dot{V}(\boldsymbol{x}) \leq-2 \alpha_{x} V(\boldsymbol{x})$. Da aus Gl. (4) folgt, dass $\underline{\alpha}_{V}(c) \leq \alpha_{x}, \forall \boldsymbol{x} \in G(V, c) \backslash\{\boldsymbol{0}\}$, folgt im Weiteren, dass $\dot{V}(\boldsymbol{x}) \leq-2 \alpha_{x} V(\boldsymbol{x}) \leq-2 \underline{\alpha}_{V}(c) V(\boldsymbol{x})$. Daraus folgt dann, dass $V(\boldsymbol{x}) \leq V\left(\boldsymbol{x}_{0}\right) e^{-2 \underline{\alpha}_{V}(\bar{c}) t}, \forall \boldsymbol{x} \in G(V, c) \backslash\{\boldsymbol{0}\}$ und schließlich mit Gl. (2), dass $\|x\| \leq \sqrt{V\left(\boldsymbol{x}_{0}\right) / \lambda_{\text {min }}(\boldsymbol{P})} e^{-\underline{\alpha}_{V}(c) t}$, d.h. dass $\underline{\alpha}_{V}(c)$ eine untere Grenze der Konvergenzrate des Systems ist.

Da verschiedene Ljapunov-Funktionen existieren, ergeben sich mehrere untere Grenzen $\underline{\alpha}_{V}(c)$. Ein Vorteil dieser gebietsabhängigen Konvergenzrate ist, dass sie unabhängig von der Systemordnung eine skalare Funktion ist. Darüber hinaus stellt diese eine untere Grenze der Konvergenzrate des Systems entlang aller Trajektorien dar, die innerhalb des jeweiligen Gebietes starten. Bildet man eine entsprechende obere Grenze der Konvergenzrate, so kann man ein gebietsabhängiges Intervall bestimmen, das alle möglichen Abklingfaktoren enthält, die entlang der Trajektorien auftreten können. Somit kann man die Performance in einem nichtlinearen Regelkreis unabhängig von der Anfangsauslenkungen untersuchen. Ein weiterer Vorteil ist, dass im Falle bestimmter Regelkreise, so wie es in diesem Beitrag der Fall ist, diese gebietsabhängige Konvergenzrate exakt bestimmt werden kann, oder zumindest auf ihre Eigenschaften untersucht werden kann, z.B. Stetigkeit, Konvexität, Monotonie etc.

Bei linearen Systemen ist $\underline{\alpha}_{V}(c)$ konstant für jedes $c>$ 0 , bei nichtlinearen Systemen variabel. Die Definition 1 für die Konvergenzrate eines nichtlinearen Systems berücksichtigt dies nicht, sodass man als untere Grenze für die Konvergenzrate des Systems für das gesamte Einzugsgebiet der Ruhelage die größte untere Schranke von $\underline{\alpha}_{V}(c)$ verwendet.

Lemma 2. Die größte untere Schranke der variablen Ljapunov-Funktion-basierten Konvergenzrate eines exponentiell stabilen nichtlinearen Systems $\dot{\boldsymbol{x}}=\boldsymbol{f}(\boldsymbol{x}), \boldsymbol{x} \in \mathbb{R}^{n}$ 
innerhalb des Einzugsgebietes der Ruhelage $\boldsymbol{x}_{\mathrm{R}}=\mathbf{0}$,

$$
\underline{\alpha}_{V}=\inf _{c>0}\left\{\underline{\alpha}_{V}(c)=\frac{1}{2} \cdot \inf _{x \in G(V, c) \backslash\{0\}}\left(-\frac{\dot{V}(\boldsymbol{x})}{V(\boldsymbol{x})}\right)\right\},
$$

stellt eine untere Grenze der Konvergenzrate $\alpha$ des nichtlinearen Systems dar.

Beweis. Der Beweis folgt unmittelbar aus dem Beweis des Lemmas 1 und wird hier weggelassen.

Diese Schranke wird zwar in vielen Fällen für den Reglerentwurf verwendet, vgl. z.B. $[8,11,12,14,15,20]$, sie ist aber kein geeignetes Maß, verschiedene Regelsysteme $\mathrm{zu}$ vergleichen, da für zwei verschiedene Regelsysteme zwei verschiedene Variationen von $\underline{\alpha}_{V}(c)$ mit der gleichen unteren Schranke $\underline{\alpha}_{V}$ erzeugt werden können. In den weiteren Abschnitten wird $\underline{\alpha}_{V}(c)$ für verschiedene Regelsysteme analysiert und für den Reglerentwurf verwendet.

\section{Sättigende lineare Regelungen}

Untersucht werden lineare und nichtlineare Regelungen für lineare Regelstrecken mit Stellgrößenbeschränkung. Es wird ohne Einschränkung der Allgemeinheit angenommen, dass die steuerbaren linearen Regelstrecken in Regelungsnormalform vorliegen, d.h.

$$
\dot{\boldsymbol{x}}=\boldsymbol{A} \boldsymbol{x}+\boldsymbol{b} u, x \in \mathbb{R}^{n}, u \in \mathbb{R},|u| \leq 1 .
$$

mit

$$
\boldsymbol{A}=\left[\begin{array}{rrrc}
0 & 1 & \cdots & 0 \\
\vdots & \vdots & \ddots & \vdots \\
0 & 0 & \cdots & 1 \\
-a_{0} & -a_{1} & \cdots & -a_{n-1}
\end{array}\right], \quad \boldsymbol{b}=\left[\begin{array}{c}
0 \\
\vdots \\
0 \\
1
\end{array}\right]
$$

Die Stabilitätsanalyse der nichtlinearen Regelkreise kann wie in [8] durch die direkte Methode von Ljapunov erfolgen, wodurch auch eine untere Grenze der Konvergenzrate angegeben werden kann. Ist darüber hinaus die lineare Regelung von der zu ermittelnden Ljapunov-Funktion abhängig, so kann man auch die resultierende Konvergenzrate des Gesamtsystems optimieren.

\subsection{Konvergenzoptimale Regelung}

Für das System (6) lautet die sättigende lineare Zustandsrückführung, welche für eine symmetrisch positiv definite Matrix $\boldsymbol{P}>\mathbf{0}$ die Konvergenzrate $\underline{\alpha}_{V}$ für jedes $\boldsymbol{x} \in$ $\mathcal{E}(\boldsymbol{P}, c) \backslash\{\mathbf{0}\}$ maximiert, [8]

$$
u=-\operatorname{sgn}\left(\boldsymbol{b}^{\top} \boldsymbol{P} \boldsymbol{x}\right) .
$$

Dies kann man wie folgt zeigen. Laut Definition 2 ist das Gebiet $\mathcal{E}(\boldsymbol{P}, c)$ für das System (6) kontraktiv invariant, wenn für eine gegebene Funktion $V(\boldsymbol{x}, u)=\boldsymbol{x}^{\top} \boldsymbol{P} \boldsymbol{x}$, mit $\boldsymbol{P}>\mathbf{0}$ und folglich für

$$
\dot{V}(\boldsymbol{x}, u)=\boldsymbol{x}^{\top}\left(\boldsymbol{A}^{\top} \boldsymbol{P}+\boldsymbol{P} \boldsymbol{A}\right) \boldsymbol{x}+2 \boldsymbol{x}^{\top} \boldsymbol{P} \boldsymbol{b} u,
$$

$\dot{V}(\boldsymbol{x}, u)<0, \forall \boldsymbol{x} \in \mathcal{E}(\boldsymbol{P}, c) \backslash\{\mathbf{0}\}$ gilt. Darüber hinaus ist $\dot{V}(\boldsymbol{x}, u)$ für jedes $\boldsymbol{x} \in \mathcal{E}(\boldsymbol{P}, c) \backslash\{\boldsymbol{0}\}$ minimal, wenn für die beschränkte Stellgröße $|u| \leq 1$ Gl. (7) gilt, d.h.

$$
\arg \min _{|u| \leq 1} \dot{V}(\boldsymbol{x}, u)=-\operatorname{sgn}\left(\boldsymbol{b}^{\top} \boldsymbol{P} \boldsymbol{x}\right) .
$$

Bemerkung 1 ([8]). Das ellipsoidale Gebiet $\mathcal{E}(\boldsymbol{P}, c)$ ist genau dann für das System (6) mit dem Regelgesetz (7) kontraktiv invariant, wenn es mit einem beschränkten Regelgesetz $|u| \leq 1$ (auch ein lineares Regelgesetz) kontraktivinvariant ist.

\subsection{Konvergenzanalyse}

Die in Gl. (4) definierte Konvergenzrate $\underline{\alpha}(\boldsymbol{P}, c):=\underline{\alpha}_{V}(c)$ wird bei dem System $\dot{\boldsymbol{x}}=\boldsymbol{A} \boldsymbol{x}-\boldsymbol{b} \operatorname{sgn}\left(\boldsymbol{b}^{\top} \boldsymbol{P} \boldsymbol{x}\right)$ auf dem Rand des Gebietes $\partial \mathcal{E}(\boldsymbol{P}, \boldsymbol{c})$ erzielt. Dieser Wert steigt zwar mit kleiner werdendem $c$, d.h. mit kleiner werdendem Abstand zur Ruhelage, ist jedoch durch die Matrix $\boldsymbol{P}$ beschränkt. Folgender Satz verdeutlicht diese Abhängigkeit:

Satz 1 ([8]). Für das System $\dot{\boldsymbol{x}}=\boldsymbol{A} \boldsymbol{x}-\boldsymbol{b} \operatorname{sgn}\left(\boldsymbol{b}^{\top} \boldsymbol{P} \boldsymbol{x}\right)$ mit den kontraktiv invarianten Gebieten $\mathcal{E}(\boldsymbol{P}, c)$ gilt

(a) $2 \underline{\alpha}(\boldsymbol{P}, c)=\frac{1}{c} \cdot \min _{\boldsymbol{x} \in \partial \mathcal{\varepsilon}(\boldsymbol{P}, c)}(-\dot{V}(\boldsymbol{x}))$,

(b) $\underline{\alpha}(\boldsymbol{P}, c)$ ist streng monoton steigend für kleiner werdendes $c$,

(c) $\lim _{c \rightarrow 0} \underline{\alpha}(\boldsymbol{P}, c)=\frac{\beta_{0}}{2} \quad$ mit

$$
\beta_{0}=\min _{\substack{\boldsymbol{x} \in \partial \mathcal{B}(\boldsymbol{P}, 1), \boldsymbol{x}^{\top} \boldsymbol{P} \boldsymbol{P}=0}}\left[-\boldsymbol{x}^{\top}\left(\boldsymbol{A}^{\top} \boldsymbol{P}+\boldsymbol{P} \boldsymbol{A}\right) \boldsymbol{x}\right] .
$$

Der Beweis des Satzes 1 kann in [8] gefunden werden. Angemerkt sei, dass innerhalb der Mannigfaltigkeit $\boldsymbol{x}^{\top} \boldsymbol{P} \boldsymbol{b}=0$ die Funktion $\dot{V}(\boldsymbol{x})$ konstant ist und folglich der Wert $\beta_{0}$ unabhängig vom betrachteten Gebiet $\partial \mathcal{E}(\boldsymbol{P}, c)$ konstant bleibt. Den Wert $\beta_{0}$ kann man gezielt wie folgt beeinflussen: aus Gl. (9) ist ersichtlich, dass $\dot{V}\left(\boldsymbol{x},-\operatorname{sgn}\left(\boldsymbol{b}^{\top} \boldsymbol{P} \boldsymbol{x}\right)\right) \leq$ $\dot{V}\left(\boldsymbol{x},-\operatorname{sat}\left(\boldsymbol{k}^{\top} \boldsymbol{x}\right)\right)$ für jedes $\boldsymbol{k} \in \mathbb{R}^{n}$ und jedes $\boldsymbol{x} \in \mathcal{E}(\boldsymbol{P}, c) \backslash\{\mathbf{0}\}$ gilt. Darüber hinaus erreicht die variable Konvergenzrate, die mit einem Regelgesetz $u=-\operatorname{sat}\left(\boldsymbol{k}^{\top} \boldsymbol{x}\right)$ erzielt wird, ihr Maximum im linearen Bereich, wo $\left|\boldsymbol{k}^{\top} \boldsymbol{x}\right| \leq 1$ gilt. Die- 
ses Maximum ist gleich ${ }^{1}-\lambda_{\max }\left\{\left[\boldsymbol{A}_{\mathrm{g}}^{\top} \boldsymbol{P}+\boldsymbol{P} \boldsymbol{A}_{\mathrm{g}}\right] \boldsymbol{P}^{-1}\right\}$, mit $\boldsymbol{A}_{\mathrm{g}}=\boldsymbol{A}-\boldsymbol{b} \boldsymbol{k}^{\top}$ und stellt eine untere Grenze der Konvergenzrate des Gesamtsystems $-\max _{i} \operatorname{Re} \lambda_{\mathrm{i}}\left(\boldsymbol{A}_{\mathrm{g}}\right)$ dar. Durch die gezielte Wahl eines Reglers $u=-\boldsymbol{k}^{\top} \boldsymbol{x}$, so dass z.B. die Eigenwerte der Systemmatrix des geschlossenen Regelkreises nach links verschoben werden, kann die Matrix $\boldsymbol{P}$ mit Hilfe des Optimierungsproblems aus Gl. (3), gefunden werden, die laut Bemerkung 1 auch für das Regelgesetz $u=-\operatorname{sgn}\left(\boldsymbol{b}^{\top} \boldsymbol{P} \boldsymbol{x}\right)$ eine Ljapunov-Matrix ist und folglich den Wert $\beta_{0}$ beeinflusst. Dies ermöglicht also die Bestimmung einer beliebigen aber endlichen maximalen Konvergenzrate $\beta_{0}$,

$$
\beta_{0}=\sup _{\boldsymbol{k}^{\top}} \min _{\boldsymbol{x} \in \partial \mathcal{E}(\boldsymbol{P}, 1)}\left[-\boldsymbol{x}^{\top}\left(\boldsymbol{A}_{\mathrm{g}}^{\top} \boldsymbol{P}+\boldsymbol{P} \boldsymbol{A}_{\mathrm{g}}\right) \boldsymbol{x}\right],
$$

jedoch auf Kosten eines immer kleiner werdenden Einzugsgebietes $\mathcal{E}(\boldsymbol{P}, \boldsymbol{c})$. Um diesem Kompromiss entgegenzuwirken, können parameterabhängige Regelgesetze verwendet werden, die die Form und Größe des Einzugsgebietes an den Abstand zur Ruhelage anpassen, z.B. [9]. Eine weitere Methode ist die weiche strukturvariable Regelung, die im nächsten Abschnitt geschildert wird.

\section{Sättigende weiche strukturvariable Regelungen mittels impliziten Ljapunov-Funktionen (implizite WSVR)}

Im Falle der strukturvariablen Regelung wird zwischen verschiedenen Regelgesetzen während des Ausregelvorgangs umgeschaltet. Dabei unterscheidet man zwischen parameter- und strukturumschaltenden Regelgesetzen. In dieser Arbeit wird eine Unterklasse der Ersteren betrachtet. Eine Übersicht solcher Regelungen bietet [3]. Die erste systematisch entwickelte Regelung dieser Art findet sich in [13]. Im Folgenden werden das Regelgesetz und die Selektionsgleichung dargestellt und der Stabilitätssatz in einer $\mathrm{zu}$ dieser Untersuchung passenderen Form wiederaufgenommen.

1 Vgl. Abschnitt 4.2 für eine detaillierte Herleitung.

2 Der Beweis der Behauptung aus Gl. (11) bezgl. des Gleichheitszeichens kann in [10] gefunden werden.

\section{Regelgesetz.}

Bei der untersuchten Regelung lautet das Regelgesetz in allgemeiner Form

$$
u=-f(x, v)
$$

wobei der Parameter $v \in \mathbb{R}$ aus einer vorgegebenen Selektionsgleichung $g(v, \boldsymbol{x})=0$ implizit oder explizit bestimmt wird. Ein Regelgesetz aus dieser Klasse ist die nichtsättigende implizite WSVR, die auf [1] zurückgeht. Es handelt sich dabei um eine vom Parameter $v$ abhängige lineare $\mathrm{Zu}$ standsrückführung

$$
u=-\boldsymbol{h}_{v}^{\top} \boldsymbol{x}
$$

wobei $\boldsymbol{h}_{v}=\boldsymbol{h}(v)$. Das Regelgesetz ist so ausgelegt, dass während des Ausregelvorgangs mit kleiner werdendem Abstand zur Ruhelage immer mehr Einfluß auf die Strecke ausgeübt wird, d.h., dass der Abstand zwischen den momentanen Eigenwerten des geschlossenen Regelkreises und denjenigen der Regelstrecke immer größer wird, aber die Stellgrößenbeschränkung nicht überschritten wird. Folglich wird der Stellgrößenbereich bei kleinen Auslenkungen von der Ruhelage im Vergleich zur linearen Regelung besser ausgenutzt. Betrachtet man den geschlossenen Regelkreis bei der Variation der Reglerverstärkung, so werden die momentanen Eigenwerte auf (vorgebbare) Bahnen wandern. Strahlenförmige Bahnen, wie in Abbildung 1 (links) gezeigt, können durch das folgende Regelgesetz [1] festgelegt werden:

$$
\begin{aligned}
\boldsymbol{h}_{v} & =\boldsymbol{D}_{v}^{-1} \hat{\boldsymbol{a}}-\boldsymbol{a}, \boldsymbol{a}^{\top}=-\left[\begin{array}{lll}
0 \cdots 0 & 1
\end{array}\right] \boldsymbol{A}, \\
\hat{\boldsymbol{a}}^{\top} & =-\left[\begin{array}{lll}
0 \cdots 0 & 1
\end{array}\right] \hat{\boldsymbol{A}}_{1}, \hat{\boldsymbol{A}}_{1}=\boldsymbol{A}-\boldsymbol{b} \boldsymbol{h}_{1}^{\top}, \\
\boldsymbol{D}_{v} & =\operatorname{diag}\left(v^{n}, v^{n-1}, \ldots, v\right) .
\end{aligned}
$$

Ähnliche Regelgesetze finden sich in [11], sowie in [15] (sättigende implizite WSVR). In dieser Arbeit wird ein weiteres Regelgesetz, angelehnt an [8], analysiert, das die maximale Konvergenzrate erzielt. Dies wird in Abschnitt 4.1 gezeigt.

\section{Selektionsgleichung.}

In der allgemeinen Form ist die Selektionsgleichung auf einer Menge

$$
H=\left\{(v, \boldsymbol{x}) \mid 0<v<\bar{v}, \boldsymbol{x} \in U_{0} \backslash\{\mathbf{0}\}\right\}
$$

definiert, wobei $U_{0}$ eine Umgebung der Ruhelage ist, und sie lautet

$$
g(v, x)=0 .
$$


Die skalare Funktion $g(v, \boldsymbol{x})$ ist stetig und differenzierbar bzgl. $v$ und $\boldsymbol{x}$, und erfüllt folgende Bedingungen:

(S1) $\lim _{v \rightarrow 0^{+}} g(\boldsymbol{x}, v)>0$ und $\lim _{v \rightarrow \bar{v}} g(\boldsymbol{x}, v)<0, \forall \boldsymbol{x} \in U_{0} \mid\{\mathbf{0}\}$,

(S2) Für $\boldsymbol{x}=\mathbf{0}$ folgt $v \rightarrow 0^{+}$.

Eine spezielle Form der Gleichung (15), die ebenfalls Bedingungen (S1) und (S2) erfüllen muss, ist

$$
g(x, v)=\boldsymbol{x}^{\top} \boldsymbol{P}_{v} \boldsymbol{x}-1=0,
$$

wobei die Matrix $\boldsymbol{P}_{v}=\boldsymbol{P}(v)$ vorerst nicht näher spezifiziert wird. Folglich erzeugt die Selektionsgleichung ellipsoidale Gebiete im Zustandsraum, die durch die vom Parameter $v$ abhängige Matrix $\boldsymbol{P}_{v}$ skaliert und gedreht werden.

Die Veränderung des Selektionsparameters $v(\boldsymbol{x})$ : $U_{0} \backslash\{\mathbf{0}\} \rightarrow(0, \bar{v}]$ ist an die Ränder der ellipsoidalen Gebiete $\partial \mathcal{E}\left(\boldsymbol{P}_{v}, 1\right) \subset \mathbb{R}^{n}$ gekoppelt. Jedem Gebiet entspricht ein eindeutiger Wert des Parameters $v \in(0, \bar{v}]$, dem äußeren Gebiet der Wert $v(\boldsymbol{x})=\bar{v}$, dem innersten Gebiet der Wert $v(\mathbf{0}) \rightarrow 0^{+}$. Abbildung 1 (rechts) veranschaulicht zwei solche Gebiete für den Fall $n=2$. Entsprechend wird während des Ausregelvorgangs für jedes $\boldsymbol{x} \in U_{0} \backslash\{\boldsymbol{0}\}$ der Wert des Parameters $v(\boldsymbol{x})$ aus der Selektionsgleichung numerisch bestimmt.

Die Gebiete sind infinitesimal dicht ineinander verschachtelt, wobei die Verschachtelung wie folgt definiert ist.

Definition 3. Zwei Gebiete $\mathcal{E}\left(\boldsymbol{P}_{1}, c_{1}\right)$ und $\mathcal{E}\left(\boldsymbol{P}_{2}, c_{2}\right)$ sind verschachtelt, wenn deren Ränder keine gemeinsamen Punkte haben, d.h. $\partial \mathcal{E}\left(\boldsymbol{P}_{1}, c_{1}\right) \cap \partial \mathcal{E}\left(\boldsymbol{P}_{2}, c_{2}\right)=\emptyset$ und wenn $\mathcal{E}\left(\boldsymbol{P}_{2}, c_{2}\right) \subset \mathcal{E}\left(\boldsymbol{P}_{1}, c_{1}\right), \forall c_{2}<c_{1}$ gilt.

Die Matrix $\boldsymbol{P}_{v}$ kann in der Form $\boldsymbol{P}_{v}=e_{v} \boldsymbol{D}_{v}^{-1} \boldsymbol{P}_{1} \boldsymbol{D}_{v}^{-1}$, mit $\boldsymbol{D}_{v}=\operatorname{diag}\left(v^{n}, \ldots, v^{2}, v\right), e_{v}=\boldsymbol{h}_{v}^{\top} \boldsymbol{P}_{v}^{-1} \boldsymbol{h}_{v}$ und $\boldsymbol{P}_{1}>\mathbf{0}$ gewählt werden, sodass sich die Überprüfung der kontraktiven Invarianz der ellipsoidalen Gebiete $\mathcal{E}\left(\boldsymbol{P}_{v}, 1\right)$ auf die des äußeren Gebietes $\mathcal{E}\left(\boldsymbol{P}_{1}, 1\right)$ reduziert. Die Skalierungsfunktion $e_{v}$ ist dabei so bestimmt, dass das jeweilige Gebiet $\mathcal{E}\left(\boldsymbol{P}_{v}, 1\right)$ unter der Bedingung

$$
\mathcal{E}\left(\boldsymbol{P}_{v}, 1\right) \subset\left\{\boldsymbol{x} \in \mathbb{R}^{n}|| \boldsymbol{h}^{\top}(v) \boldsymbol{x} \mid \leq 1\right\}=: \mathcal{L}(v)
$$

maximiert wird. Die Gebiete $\mathcal{L}(v)$ werden ebenfalls im $\mathrm{Ab}$ bildung 1 (rechts) gezeigt.

Die Selektionsgleichung kann man vereinfachen [12], indem man die Skalierungsfunktion $e(v)$ weglässt, d.h.

$$
\boldsymbol{P}_{v}=\boldsymbol{D}_{v}^{-1} \boldsymbol{P}_{1} \boldsymbol{D}_{v}^{-1} .
$$

Durch diese Vereinfachung kann man zwar auch Regler für instabile Strecken entwerfen [12], es ist jedoch nicht mehr
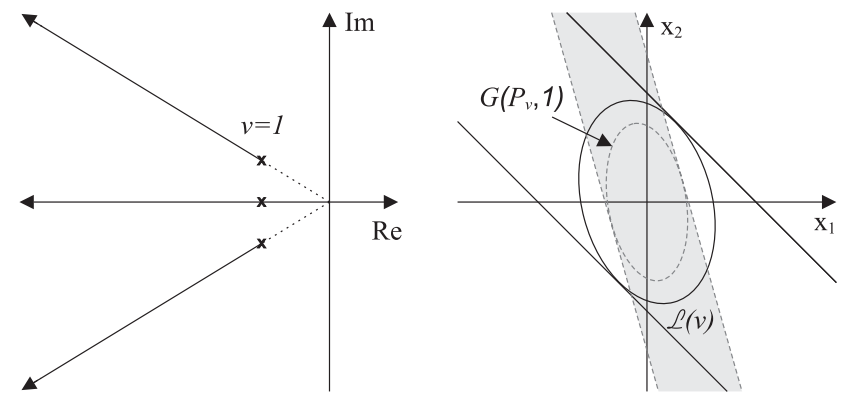

Abbildung 1: Momentane Eigenwerte (links) und kontraktiv invariante Gebiete (rechts) für den Fall $x \in \mathbb{R}^{2}$, mit $v \in(0,1]$.

sichergestellt, dass die Stellgröße die Beschränkung nicht überschreitet. Dies muss durch eine zusätzliche Bedingung beim Regelungsentwurf sichergestellt werden. Weitere Formen für die Matrix $\boldsymbol{P}_{v}$ finden sich z.B. in $[9,16]$.

\section{Stabilitätsnachweis.}

Die Stabilität des Gesamtsystems mit dem allgemeinen Regelgesetz (12) und Selektionsgleichung (15) wird dabei durch den Parameter $v(\boldsymbol{x})$ gewährleistet, der eine implizite Ljapunov-Funktion des Gesamtsystems darstellt. Folgendes Lemma nach [2] verdeutlicht dies.

Lemma 3 (Nach [2]). Für eine lineare Strecke in Regelungsnormalform

$$
\dot{\boldsymbol{x}}=\boldsymbol{A} \boldsymbol{x}+\boldsymbol{b} u, x \in \mathbb{R}^{n}, u \in \mathbb{R},|u| \leq 1,
$$

mit dem Regelgesetz $u=-f(\boldsymbol{x}, v)$ und der Selektionsgleichung $g(x, v)=0$, welche Bedingungen (S1) und (S2) erfüllt, sind die Gebiete

$$
G(v)=\left\{\boldsymbol{x} \in U_{0} \backslash\{\mathbf{0}\} \mid g(\boldsymbol{x}, v)<0\right\},
$$

für $v \in(0, \bar{v}]$ ineinander verschachtelt und kontraktiv invariant, wenn die Bedingungen

$$
\begin{gathered}
(S 3)-\infty<\frac{\partial g(v, \boldsymbol{x})}{\partial v}<0, \\
(S 4) \quad \frac{\partial g(v, \boldsymbol{x}(t))}{\partial t}<0
\end{gathered}
$$

$\forall(v, \boldsymbol{x}) \in H$ erfüllt sind. Im Weiteren ist die Funktion $0<$ $v(\boldsymbol{x}) \leq \bar{v}$, welche durch die Gleichung $g(v, \boldsymbol{x})=0$ implizit definiert ist und in $\boldsymbol{x}=\mathbf{0}$ durch $v(\mathbf{0})=0$ stetig erweiterbar ist, eine Ljapunov-Funktion des Systems. 


\subsection{Konvergenzoptimale implizite WSVR}

Für die Selektionsgleichung $\boldsymbol{x}^{\top} \boldsymbol{P}_{v} \boldsymbol{x}-1=0$ aus Gl. (16) folgt mit $\dot{v}(\boldsymbol{x})=-\frac{\partial g(x, v) / \partial t}{\partial g(x, v) / \partial v}$, dass

$$
\dot{v}(\boldsymbol{x})=-\frac{\boldsymbol{x}^{\top}\left(\boldsymbol{A}^{\top} \boldsymbol{P}_{v}+\boldsymbol{P}_{v} \boldsymbol{A}\right) \boldsymbol{x}+2 \boldsymbol{x}^{\top} \boldsymbol{P}_{v} \boldsymbol{b} \cdot u}{\boldsymbol{x}^{\top} \boldsymbol{P}_{v}^{\prime} \boldsymbol{x}}
$$

mit $\boldsymbol{P}_{v}^{\prime}=\partial \boldsymbol{P}_{v} / \partial v$ gilt. Folglich gilt

$$
\arg \min _{|u| \leq 1} \dot{v}(\boldsymbol{x})=-\operatorname{sgn}\left(\boldsymbol{b}^{\top} \boldsymbol{P}_{v} \boldsymbol{x}\right)
$$

weil $-\boldsymbol{x}^{\top} \boldsymbol{P}_{v} \boldsymbol{b} \cdot \operatorname{sgn}\left(\boldsymbol{b}^{\top} \boldsymbol{P}_{v} \boldsymbol{x}\right)=-\left|\boldsymbol{x}^{\top} \boldsymbol{P}_{v} \boldsymbol{b}\right|$ die maximal mögliche Abnahme der Funktion $v(\boldsymbol{x})$ in einem Zustandspunkt $\boldsymbol{x} \in \partial \mathcal{E}\left(\boldsymbol{P}_{v}, 1\right)$ unter der Bedingung $|u| \leq 1$ erzielt. Die sättigende implizite WSVR mit der parameterabhängigen $\mathrm{Zu}$ standsrückführung

$$
u=-\operatorname{sgn}\left(\boldsymbol{b}^{\top} \boldsymbol{P}_{v} \boldsymbol{x}\right)
$$

maximiert folglich die Konvergenzrate für jedes $v(\boldsymbol{x})$, d.h. auf dem Rand jedes Ellipsoids $\partial \mathcal{E}\left(\boldsymbol{P}_{v}, 1\right)$.

Die Verschiebung der momentanen Eigenwerte ist nicht mehr auf vordefinierte Bahnen beschränkt, die Konvergenzrate steigt jedoch durch die Auswahl einer geeigneten Matrix $\boldsymbol{P}_{v}$ mit kleiner werdendem Abstand zur Ruhelage, wie es in Abschnitt 4.2 gezeigt wird.

Folgende Sätze geben die Bedingungen für die kontraktive Invarianz der Gebiete $\mathcal{E}\left(\boldsymbol{P}_{v}, 1\right)$ für das Gesamtsystem $\dot{\boldsymbol{x}}=\boldsymbol{A} \boldsymbol{x}-\boldsymbol{b} \operatorname{sgn}\left(\boldsymbol{b}^{\top} \boldsymbol{P}_{v} \boldsymbol{x}\right)$ an:

Lemma 4. Falls die Bedingungen (S3) und (S4) aus Lemma 3 für das System $\dot{\boldsymbol{x}}=\boldsymbol{A} \boldsymbol{x}-\boldsymbol{b} f(\boldsymbol{x}, v)$, mit einem beliebigen beschränkten Regelgesetz $|f(\boldsymbol{x}, v)| \leq 1$ und einer Selektionsstrategie $g_{f}(\boldsymbol{x}, v)=\boldsymbol{x}^{\top} \boldsymbol{P}_{v} \boldsymbol{x}-1=0, v \in(0, \bar{v}]$ erfüllt sind, so sind diese immer auch für das System $\dot{\boldsymbol{x}}=\boldsymbol{A x}-$ $\boldsymbol{b} \operatorname{sgn}\left(\boldsymbol{b}^{\top} \boldsymbol{P}_{v} \boldsymbol{x}\right)$ mit $g_{\mathrm{s}}(\boldsymbol{x}, v)=\boldsymbol{x}^{\top} \boldsymbol{P}_{v} \boldsymbol{x}-1=0$, mit $v \in(0, \bar{v}]$ erfüllt.

Beweis. Wenn die Bedingungen (S3) und (S4) für das System $\dot{\boldsymbol{x}}=\boldsymbol{A} \boldsymbol{x}-\boldsymbol{b} f(\boldsymbol{x}, v)$, mit $g(\boldsymbol{x}, v)=\boldsymbol{x}^{\top} \boldsymbol{P}_{v} \boldsymbol{x}-1=0, v \in$ $(0, \bar{v}]$ und $|f(x, v)| \leq 1$ erfüllt sind, dann folgt aus Lemma 3, dass die Gebiete $\mathcal{E}\left(\boldsymbol{P}_{v}, 1\right)$ ineinander verschachtelt und kontraktiv invariant für das System sind. Außerdem gilt

$$
-\infty<\frac{\partial g_{f}(\boldsymbol{x}, v)}{\partial v}=\boldsymbol{x}^{\top} \boldsymbol{P}_{v}^{\prime} \boldsymbol{x}=\frac{\partial g_{s}(\boldsymbol{x}, v)}{\partial v}<0, \forall(\boldsymbol{x}, v) \in H
$$

$$
\begin{aligned}
& \frac{\partial g_{s}(\boldsymbol{x}(t), v)}{\partial t}=\boldsymbol{x}^{\top}\left(A^{\top} \boldsymbol{P}_{v}+\boldsymbol{P}_{v} \boldsymbol{A}\right) \boldsymbol{x}-2\left|\boldsymbol{x}^{\top} \boldsymbol{P}_{v} \boldsymbol{b}\right| \\
& =\frac{\partial g_{f}(\boldsymbol{x}(t), v)}{\partial t}+2 \boldsymbol{x}^{\top} \boldsymbol{P}_{v} \boldsymbol{b} f(\boldsymbol{x}, v)-2\left|\boldsymbol{x}^{\top} \boldsymbol{P}_{v} \boldsymbol{b}\right| \\
& =\frac{\partial g_{f}(\boldsymbol{x}(t), v)}{\partial t}+2\left|\boldsymbol{x}^{\top} \boldsymbol{P}_{v} \boldsymbol{b}\right|\left[\operatorname{sgn}\left(\boldsymbol{x}^{\top} \boldsymbol{P}_{v} \boldsymbol{b}\right) f(\boldsymbol{x}, v)-1\right] \\
& =\frac{\partial g_{f}(\boldsymbol{x}, v)}{\partial t}+
\end{aligned}
$$

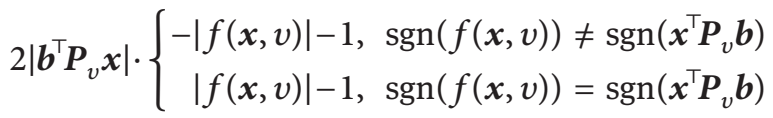

$$
\begin{aligned}
& <0, \forall(x, v) \in H \text {. }
\end{aligned}
$$

Somit folgt, dass die Gebiete $\mathcal{E}\left(\boldsymbol{P}_{v}, 1\right)$ ineinander verschachtelt und kontraktiv invariant auch für das System $\dot{\boldsymbol{x}}=\boldsymbol{A} \boldsymbol{x}-\boldsymbol{b} \operatorname{sgn}\left(\boldsymbol{b}^{\top} \boldsymbol{P}_{v} \boldsymbol{x}\right)$ mit $g_{s}(\boldsymbol{x}, v)=\boldsymbol{x}^{\top} \boldsymbol{P}_{v} \boldsymbol{x}-1=0$ und $v \in(0, \bar{v}]$ sind.

Im Folgenden zeigt Satz 2 drei äquivalente Aussagen, die zum Entwurf des konvergenzoptimalen Regelgesetzes verwendet werden.

Satz 2. Gegeben sei die lineare Strecke in Regelungsnormalform

$$
\dot{\boldsymbol{x}}=\boldsymbol{A} \boldsymbol{x}+\boldsymbol{b} u, x \in \mathbb{R}^{n}, u \in \mathbb{R},|u| \leq 1,
$$

die in $v$ und $\boldsymbol{x}$ stetige und stetig differenzierbare Funktion $g_{u}(\boldsymbol{x}, v)$ und die Gleichung

$$
g_{u}(\boldsymbol{x}, v)=\boldsymbol{x}^{\top} \boldsymbol{P}_{v} \boldsymbol{x}-1=0,
$$

mit $v \in[v, \bar{v}]$, sodass Bedingungen (S1) und (S2) erfüllt sind. Folgende Aussagen sind äquivalent:

(a) Es existiert ein Regelgesetz $u_{f}=-f(x, v)$, mit $\left|u_{f}\right| \leq 1$, welches die Bedingungen (S3) und (S4) für das System (22) mit der Selektionsgleichung (23) erfüllt.

(b) Die Bedingungen (S3) und (S4) sind für das System (22) mit dem Regelgesetz $u_{s}=-\operatorname{sgn}\left(\boldsymbol{b}^{\top} \boldsymbol{P}_{v} \boldsymbol{x}\right)$ und Selektionsgleichung (23) erfüllt.

(c) Es existiert eine Funktion $\kappa_{v}:[\underline{v}, \bar{v}] \rightarrow \mathbb{R}_{+}$, sodass die Bedingungen (S3) und (S4) für das System (22) mit dem Regelgesetz $u_{\kappa_{v}}=-\operatorname{sat}\left(\kappa_{v} \boldsymbol{b}^{\top} \boldsymbol{P}_{v} \boldsymbol{x}\right)$ und Selektionsgleichung (23) erfüllt sind.

Bemerkung 2. Die vom Parameter $v$ abhängige Matrix $\boldsymbol{P}_{v}$ aus der Selektionsgleichung (23) und das Regelgesetz $f(x, v)$ aus Punkt $(a)$ sind im obigen Satz nicht weiter spezifiziert. Die Selektionsgleichungen und Regelgesetze aus $[1,11,12,14-16]$ können somit verwendet werden.

Beweis. $(a) \Rightarrow(b)$ Wenn (a) gilt, dann folgt aus Lemma 4, dass die Bedingungen (S3) und (S4) für das System (22) mit dem Regelgesetz $u_{\mathrm{s}}=-\operatorname{sgn}\left(\boldsymbol{b}^{\top} \boldsymbol{P}_{v} \boldsymbol{x}\right)$ und der Selektionsgleichung (23) erfüllt sind, d.h. es folgt $(b)$. 
$(b) \Rightarrow(c)$ Aus $(b)$ folgt, dass

$$
\begin{aligned}
\frac{\partial g_{u_{s}}(\boldsymbol{x}, v)}{\partial t}= & \boldsymbol{x}^{\top}\left(\boldsymbol{A}^{\top} \boldsymbol{P}_{v}+\boldsymbol{P}_{v} \boldsymbol{A}\right) \boldsymbol{x}-2 \boldsymbol{x}^{\top} \boldsymbol{P}_{v} \boldsymbol{b} \operatorname{sgn}\left(\boldsymbol{b}^{\top} \boldsymbol{P}_{v} \boldsymbol{x}\right) \\
& <0, \forall \boldsymbol{x} \in \partial \mathcal{E}\left(\boldsymbol{P}_{v}, 1\right) .
\end{aligned}
$$

Angenommen sei im Weiteren, dass $\boldsymbol{b}^{\top} \boldsymbol{P}_{v}=\left[\begin{array}{ll}\mathbf{0} & r v^{-2}\end{array}\right]$ mit $r>0$. Ist dies nicht der Fall, so kann man für jedes $v$ eine Zustandstransformation $\tilde{\boldsymbol{x}}=\boldsymbol{T}_{v} \boldsymbol{x}$, mit $\boldsymbol{T}_{v}$ nichtsingulär finden, sodass $\tilde{\boldsymbol{P}}_{v}=\left(\boldsymbol{T}_{v}^{-1}\right)^{\top} \boldsymbol{P}_{v} \boldsymbol{T}_{v}^{-1}>0,{ }^{3} \tilde{\boldsymbol{b}}=\boldsymbol{T}_{v} \boldsymbol{b}$ und $\tilde{\boldsymbol{b}}^{\top} \tilde{\boldsymbol{P}}_{v}=\left[\begin{array}{ll}\mathbf{0} & \tilde{p}_{(n, n)} v^{-2}\end{array}\right]$ mit $\tilde{p}_{(n, n)}>0$.

Die Matrix $\left(\boldsymbol{A}^{\top} \boldsymbol{P}_{v}+\boldsymbol{P}_{v} \boldsymbol{A}\right)-2 \kappa_{v} \boldsymbol{P}_{v} \boldsymbol{b} \boldsymbol{b}^{\top} \boldsymbol{P}_{v}$ lässt sich dann wie folgt in eine Blockmatrix zerlegen

$$
\begin{aligned}
\mathcal{A} & =\underbrace{\left[\begin{array}{cc}
\boldsymbol{Q}_{v, 1} & \boldsymbol{q}_{v, 12} \\
\boldsymbol{q}_{v, 12}^{\top} & q_{v, 2}
\end{array}\right]}_{A^{\top} \boldsymbol{P}_{v}+\boldsymbol{P}_{v} A}-2 \kappa_{v} \underbrace{\left[\begin{array}{cc}
\mathbf{0} & \mathbf{0} \\
\mathbf{0}^{\top} & \left(r v^{-2}\right)^{2}
\end{array}\right]}_{\boldsymbol{P}_{v} \boldsymbol{b} \boldsymbol{b}^{\top} \boldsymbol{P}_{v}} \\
& =\left[\begin{array}{cc}
\boldsymbol{Q}_{v, 1} & \boldsymbol{q}_{v, 12} \\
\boldsymbol{q}_{v, 12}^{\top} & q_{v, 2}-2 \kappa_{v}\left(r v^{-2}\right)^{2}
\end{array}\right] .
\end{aligned}
$$

Für jedes $\boldsymbol{x}^{\top}=\left[\begin{array}{ll}\boldsymbol{x}_{1} & 0\end{array}\right]$ folgt $\boldsymbol{x}^{\top} \boldsymbol{P}_{v} \boldsymbol{b}=0$ und aus Gl. (24) $\boldsymbol{x}^{\top}\left(\boldsymbol{A}^{\top} \boldsymbol{P}_{v}+\boldsymbol{P}_{v} \boldsymbol{A}\right) \boldsymbol{x}=\boldsymbol{x}_{1}^{\top} \boldsymbol{Q}_{v, 1} \boldsymbol{x}_{1}<0$, d.h. $\boldsymbol{Q}_{v, 1} \prec 0$. Wenn eine Funktion $\kappa_{v}:[\underline{v}, \bar{v}] \rightarrow \mathbb{R}_{+}$existiert, sodass

$$
\boldsymbol{Q}_{v, 1} \mid \mathcal{A}=q_{v, 2}-2 \kappa_{v}\left(r v^{-2}\right)^{2}-\boldsymbol{q}_{v, 12}^{\top} \boldsymbol{Q}_{v, 1}^{-1} \boldsymbol{q}_{v, 12}<0
$$

gilt, folgt mit $\boldsymbol{Q}_{v, 1} \prec 0$, dass $\mathcal{A} \prec 0$ ist. Damit Gl. (26) erfüllt ist, muss

$$
\begin{aligned}
\kappa_{v}> & \frac{q_{v, 2}-\boldsymbol{q}_{v, 12}^{\top} \boldsymbol{Q}_{v, 1}^{-1} \boldsymbol{q}_{v, 12}}{2\left(r v^{-2}\right)^{2}} \\
& =\frac{\sum_{k=1}^{n} v^{-n+k-2} p_{(k, n)} \boldsymbol{a}_{(k, n)}}{r^{2} v^{-4}}-\frac{\boldsymbol{q}_{v, 12}^{\top} \boldsymbol{Q}_{v, 1}^{-1} \boldsymbol{q}_{v, 12}}{2 r^{2} v^{-4}} \\
& =\frac{a_{(n, n)} v^{2}}{r}-\frac{\boldsymbol{q}_{v, 12}^{\top} \boldsymbol{Q}_{v, 1}^{-1} \boldsymbol{q}_{v, 12}}{2 r^{2} v^{-4}},
\end{aligned}
$$

gelten, wobei $a_{(i, j)}$ das Element $(i, j)$ der Matrix $\boldsymbol{A}$ und $p_{(i, j)}$ das Element $(i, j)$ der Matrix $\boldsymbol{P}_{1}$ darstellen, sowie $p_{(i, n)}=$ $0, \forall i \in\{1, \ldots, n-1\}$ und $p_{(n, n)}=r>0$ sind. Gl. (27) ist z.B. erfüllt wenn

$$
\kappa_{v} \geq \frac{\left|a_{(n, n)}\right|}{2 r}+\frac{\lambda_{\max }\left(-\boldsymbol{Q}_{v, 1}^{-1}\right) \boldsymbol{q}_{v, 12}^{\top} \boldsymbol{q}_{v, 12}}{2 r^{2} v^{-4}}
$$

gilt, weil $\boldsymbol{q}_{v}^{\top}\left(-\boldsymbol{Q}_{v}^{-1}\right) \boldsymbol{q}_{v} \leq \lambda_{\max }\left(-\boldsymbol{Q}_{v}^{-1}\right) \boldsymbol{q}_{v}^{\top} \boldsymbol{q}_{v}, \forall \boldsymbol{q} \in \mathbb{R}^{n}$, mit $\boldsymbol{q} \neq$ $\mathbf{0}$ ist. Dabei stellt $\boldsymbol{q}_{v, 12}^{\top} \boldsymbol{q}_{v, 12}$ ein Polynom in $v$ mit dem minimalen Exponent $-4 n+2$ dar. Der maximale Wert der Funktion auf der rechten Seite von Gl. (28) kann für $v \in[\underline{v}, \bar{v}]$ numerisch bestimmt werden.

3 Bezüglich der positiven Definitheit der transformierten Matrix $\tilde{\boldsymbol{P}}_{1}$ siehe [4], Fakt 8.1.3.
Daraus resultiert, dass die Bedingungen (S3) und (S4) für das System (22) mit dem Regelgesetz $u=-\kappa_{v} \boldsymbol{b}^{\top} \boldsymbol{P}_{v} \boldsymbol{x}$ (ohne Sättigungsglied) und der Selektionsgleichung (23) erfüllt sind. Da es auch ein beschränktes Regelgesetz, hier $u_{s}$, gibt, das die Bedingungen (S3) und (S4) erfüllt, folgt, dass $\frac{\partial g_{u_{\kappa_{v}}}(x, v)}{\partial t}<0$ gilt ${ }^{4}$.

Da noch

$$
-\infty<\frac{\partial g_{u_{\kappa_{v}}}(\boldsymbol{x}, v)}{\partial v}=\frac{\partial g_{u_{\mathrm{s}}}(\boldsymbol{x}, v)}{\partial v}<0
$$

gilt, folgt schließlich aus Lemma 3 wiederum $(c)$.

$(c) \Rightarrow(a)$ Das Regelgesetz aus $(c)$ ist offensichtlich ein beschränktes Regelgesetz, das die Bedingungen (S3) und (S4) für das System (22) mit der Selektionsgleichung (23) erfüllt, d.h. es folgt $(a)$.

Satz 2 ermöglicht also auch den Entwurf eines kontinuierlichen Regelgesetzes $u_{\kappa_{v}}=-\operatorname{sat}\left(\kappa_{v} \boldsymbol{b} \boldsymbol{P}_{v} \boldsymbol{x}\right)$, das für eine hinreichend große Funktion $\kappa_{v}$ die Konvergenzrate des Systems $\dot{\boldsymbol{x}}=\boldsymbol{A}-\boldsymbol{b} \operatorname{sgn}\left(\boldsymbol{b}^{\top} \boldsymbol{P}_{v} \boldsymbol{x}\right)$ annähert.

\subsection{Konvergenzanalyse}

\section{Nichtsättigende implizite WSVR.}

Die Konvergenzrate des Systems $\dot{\boldsymbol{x}}=\hat{A}_{v} \boldsymbol{x}$, mit der Systemmatrix festgelegt in Gl. (14), d.h.

$$
\hat{A}_{v}=\boldsymbol{A}-\boldsymbol{b} \boldsymbol{h}_{v}^{\top}=\frac{1}{v} \boldsymbol{D}_{v} \hat{A}_{1} \boldsymbol{D}_{v}^{-1},
$$

kann man mit Hilfe des Lagrange-MultiplikatorenAnsatzes analysieren. Im Zusammenhang mit der Konvergenzrate der Zustandsnorm $\|x\|$ kann die Konvergenzrate der quadratischen Funktion $W(\boldsymbol{x}, v):=\boldsymbol{x}^{\top} \boldsymbol{P}_{v} \boldsymbol{x}$ untersucht werden. Folglich bildet der kleinste Abklingfaktor der Funktion $W(\boldsymbol{x}, v)$ eine untere Grenze der Konvergenzrate der Zustandsnorm $\|x\|$, d.h.

$$
\begin{aligned}
2 \underline{\alpha}_{W}(v) & =\min _{\boldsymbol{x} \in \partial \mathcal{E}\left(\boldsymbol{P}_{v}, 1\right)}\left(-\frac{\dot{W}(\boldsymbol{x}, v)}{W(\boldsymbol{x}, v)}\right) \\
& =\min _{\boldsymbol{x} \in \partial \mathcal{E}\left(\boldsymbol{P}_{v}, 1\right)}\left[-\boldsymbol{x}^{\top}\left(\hat{\boldsymbol{A}}_{v}^{\top} \boldsymbol{P}_{v}+\boldsymbol{P}_{v} \hat{\boldsymbol{A}}_{v}\right) \boldsymbol{x}\right] .
\end{aligned}
$$

Die Nebenbedingung, dass nur Zustandsvektoren auf dem Ellipsoid betrachtet werden, d.h. $\boldsymbol{x} \in \partial \mathcal{E}\left(\boldsymbol{P}_{v}, 1\right)$, kann man mit Hilfe des Lagrange-MultiplikatorenAnsatzes in die Zielfunktion einbeziehen und das Ersatzoptimierungsproblem ohne Nebenbedingung lösen. Es

4 Vgl. [14], Satz 1. 
ergibt sich

$$
\begin{aligned}
2 \underline{\alpha}_{W}(v) & =\min _{\boldsymbol{x} \neq \mathbf{0}} \underbrace{\left[-\boldsymbol{x}^{\top}\left(\hat{\boldsymbol{A}}_{v}^{\top} \boldsymbol{P}_{v}+\boldsymbol{P}_{v} \hat{\boldsymbol{A}}_{v}\right) \boldsymbol{x}+l_{v}\left(\boldsymbol{x}^{\top} \boldsymbol{P}_{v} \boldsymbol{x}-1\right)\right]}_{L\left(\boldsymbol{x}, l_{v}\right)} \\
& =-\lambda_{\max }\left[\left(\hat{\boldsymbol{A}}_{v}^{\top} \boldsymbol{P}_{v}+\boldsymbol{P}_{v} \hat{\boldsymbol{A}}_{v}\right) \boldsymbol{P}_{v}^{-1}\right] .
\end{aligned}
$$

Dabei ist $l_{v}$ der Lagrange-Operator des Optimierungsproblems. Der minimale Wert der Funktion aus Gl. (29) folgt aus der Optimalitätsbedingungen für die LagrangeFunktion $L\left(\boldsymbol{x}, l_{v}\right)$,

$$
\begin{aligned}
& \partial L\left(\boldsymbol{x}, l_{v}\right) / \partial \boldsymbol{x}=0 \Rightarrow\left(\hat{A}_{v}^{\top} \boldsymbol{P}_{v}+\boldsymbol{P}_{v} \hat{A}_{v}-l_{v} \boldsymbol{P}_{v}\right) \boldsymbol{x}=\mathbf{0}, \\
& \partial L\left(\boldsymbol{x}, l_{v}\right) / \partial l_{v}=0 \Rightarrow \boldsymbol{x}^{\top} \boldsymbol{P}_{v} \boldsymbol{x}-1=0 .
\end{aligned}
$$

Gl. (30) stellt ein allgemeines Eigenwertproblem mit der Lösung $l_{v}=\lambda\left[\left(\hat{A}_{v}^{\top} \boldsymbol{P}_{v}+\boldsymbol{P}_{v} \hat{A}_{v}\right) \boldsymbol{P}_{v}^{-1}\right]$ dar. Da die Matrizen $\hat{\boldsymbol{A}}_{v}^{\top} \boldsymbol{P}_{v}+\boldsymbol{P}_{v} \hat{\boldsymbol{A}}_{v}$ symmetrisch und $\boldsymbol{P}_{v}>0$ positiv definit sind, folgt ${ }^{5}$, dass $\operatorname{Spec}\left[\left(\hat{\boldsymbol{A}}_{\mathrm{v}}^{\top} \boldsymbol{P}_{\mathrm{v}}+\boldsymbol{P}_{\mathrm{v}} \hat{\boldsymbol{A}}_{\mathrm{v}}\right) \boldsymbol{P}_{\mathrm{v}}^{-1}\right] \subset \mathbb{R}$. Da $\hat{A}_{v}^{\top} \boldsymbol{P}_{v}+\boldsymbol{P}_{v} \hat{A}_{v} \prec 0$, folgt ${ }^{6}$ darüber hinaus, dass $\lambda\left[\left(\hat{A}_{v}^{\top} \boldsymbol{P}_{v}+\right.\right.$ $\left.\left.\boldsymbol{P}_{v} \hat{A}_{v}\right) \boldsymbol{P}_{v}^{-1}\right] \leq 0$.

Für $\boldsymbol{P}_{v}=D_{v}^{-1} \boldsymbol{P}_{1} \boldsymbol{D}_{v}^{-1}$ und $\hat{A}_{v}=\frac{1}{v} \boldsymbol{D}_{v} \hat{A}_{1} \boldsymbol{D}_{v}^{-1}$ folgt schließlich, dass

$$
\begin{aligned}
2 \underline{\alpha}_{W}(v) & =-\frac{1}{v} \lambda_{\max }\left[\boldsymbol{D}_{v}^{-1}\left(\hat{A}_{1}^{\top} \boldsymbol{P}_{1}+\boldsymbol{P}_{1} \hat{A}_{1}\right) \boldsymbol{P}_{1}^{-1} \boldsymbol{D}_{v}\right] \\
& =-\frac{1}{v} \lambda_{\max }\left[\left(\hat{A}_{1}^{\top} \boldsymbol{P}_{1}+\boldsymbol{P}_{1} \hat{A}_{1}\right) \boldsymbol{P}_{1}^{-1}\right] .
\end{aligned}
$$

Das letzte Ergebnis beruht auf der Tatsache, dass die Matrizen $\boldsymbol{D}_{v}^{-1}\left(\hat{\boldsymbol{A}}_{1}^{\top} \boldsymbol{P}_{1}+\boldsymbol{P}_{1} \hat{\boldsymbol{A}}_{1}\right) \boldsymbol{P}_{1}^{-1} \boldsymbol{D}_{v}$ und $\left(\hat{\boldsymbol{A}}_{1}^{\top} \boldsymbol{P}_{1}+\boldsymbol{P}_{1} \hat{\boldsymbol{A}}_{1}\right) \boldsymbol{P}_{1}^{-1} \ddot{a} h n-$ lich sind und folglich gleiche Eigenwerte haben. Aus Gl. (32) ist ersichtlich, dass mit kleiner werdendem $v$ die Konvergenzrate $\underline{\alpha}_{W}(v)$ steigt. Da die Gebiete ineinander verschachtelt sind, folgt auch, dass innerhalb eines Einzugsgebietes $\mathcal{E}\left(\boldsymbol{P}_{v}, 1\right)$ die definitionsgemäß minimale Konvergenzrate auf dem Rand des Gebietes erzielt wird.

Bemerkung 3. Wird die Matrix $\boldsymbol{P}_{1}$ so bestimmt, dass die Abklingrate der Funktion $W(\boldsymbol{x}, 1)$, d.h. $-\dot{W}(\boldsymbol{x}, 1)$, maximiert wird, so ist $\underline{\alpha}_{W}(v)$ für jedes $v$ maximal und bis auf das Minuszeichen gleich dem größten Realteil unter den momentanen Eigenwerten der Matrix $\hat{A}$ (vgl. Abbildung 1 links), d.h.

$$
\underline{\alpha}_{W}(v)=-\operatorname{Re} \lambda_{\max }\left(\hat{A}_{v}\right)=-\frac{1}{v} \operatorname{Re} \lambda_{\max }\left(\hat{A}_{1}\right) .
$$

Folgender Satz fasst diese Ergebnisse zusammen:

5 Vgl. [17], Fakt 6.52.

6 Vgl. [4], Fakt 8.3.7.
Satz 3 (Konvergenzrate der nichtsättigenden impliziten WSVR, Für den geschlossenen Regelkreis $\dot{\boldsymbol{x}}=\left(\boldsymbol{A}-\boldsymbol{b} \boldsymbol{k}_{v}^{\top}\right) \boldsymbol{x}=$ $\hat{A}_{v} \boldsymbol{x}$, mit $\boldsymbol{k}_{v}=\boldsymbol{D}_{v}^{-1} \hat{\boldsymbol{a}}-\boldsymbol{a}, \boldsymbol{a}=-\left[\begin{array}{llll}0 & \cdots & 0 & 1\end{array}\right] \boldsymbol{A}, \hat{\boldsymbol{a}}=$ $-\left[\begin{array}{llll}0 & \cdots & 0 & 1\end{array}\right] \hat{A}_{1}, D_{v}=\operatorname{diag}\left(v^{n}, v^{n-1}, \ldots, v\right), v \in(0,1]$ und den kontraktiv invarianten und verschachtelten Gebieten

$$
\begin{aligned}
\mathcal{E}\left(\boldsymbol{P}_{v}, 1\right)=\{\boldsymbol{x} & \left.\in U_{0} \backslash\{\boldsymbol{0}\} \mid \boldsymbol{x}^{\top} \boldsymbol{P}_{v} \boldsymbol{x}<1\right\} \\
& \subset\left\{\boldsymbol{x} \in U_{0} \backslash\{\boldsymbol{0}\}|| \boldsymbol{k}_{v}^{\top} \boldsymbol{x} \mid \leq 1\right\},
\end{aligned}
$$

mit $\boldsymbol{P}_{v}=\boldsymbol{D}_{v}^{-1} \boldsymbol{P}_{1} \boldsymbol{D}_{v}^{-1}$, gilt:

$$
\begin{aligned}
& \text { (a) } 2 \underline{\alpha}_{W}(v)=-\frac{1}{v} \lambda_{\max }\left[\left(\hat{A}_{1}^{\top} \boldsymbol{P}_{1}+\boldsymbol{P}_{1} \hat{A}_{1}\right) \boldsymbol{P}_{1}^{-1}\right], \\
& \text { (b) } \underline{\alpha}_{W}(v) \text { ist streng monoton steigend, } \\
& \text { mit sinkendem } v \\
& \text { (c) } \lim _{v \rightarrow 0^{+}} \underline{\alpha}_{W}(v)=\infty .
\end{aligned}
$$

\section{Sättigende implizite WSVR.}

Die Konvergenzrate des Systems $\dot{\boldsymbol{x}}=\boldsymbol{A} \boldsymbol{x}-\boldsymbol{b} \operatorname{sgn}\left(\boldsymbol{b}^{\top} \boldsymbol{P}_{v} \boldsymbol{x}\right)$ kann man, wie auch in [10] dargestellt, mit Hilfe des Lagrange-Multiplikatoren-Ansatzes analysieren. Untersucht wird der kleinste Abklingfaktor der Funktion $W(\boldsymbol{x}, v)$ auf jedem Ellipsoid $\partial \mathcal{E}\left(\boldsymbol{P}_{v}, 1\right)$ für $\boldsymbol{x}^{\top} \boldsymbol{P}_{v} \boldsymbol{b} \geq 0,{ }^{7}$ d.h.

$$
\begin{aligned}
& 2 \underline{\alpha}_{W}(v)=\min _{\boldsymbol{x} \in \partial \mathcal{E}\left(\boldsymbol{P}_{v}, 1\right)}\left(-\frac{\dot{W}(\boldsymbol{x}, v)}{W(\boldsymbol{x}, v)}\right) \\
& =\min _{x \in \partial \mathcal{E}\left(\boldsymbol{P}_{v}, 1\right)}(-\dot{W}(\boldsymbol{x}, v)) \\
& =\min _{x \in \partial \mathcal{E}\left(\boldsymbol{P}_{v}, 1\right)}-\left(\boldsymbol{x}^{\top}\left(\boldsymbol{A}^{\top} \boldsymbol{P}_{v}+\boldsymbol{P}_{v} \boldsymbol{A}\right) \boldsymbol{x}-2 \boldsymbol{b}^{\top} \boldsymbol{P}_{v} \boldsymbol{x}\right) \\
& =\min _{\boldsymbol{x}^{\top} \boldsymbol{P}_{v} b \geq 0}\left[-\boldsymbol{x}^{\top}\left(\boldsymbol{A}^{\top} \boldsymbol{P}_{v}+\boldsymbol{P}_{v} \boldsymbol{A}\right) \boldsymbol{x}+2 \boldsymbol{b}^{\top} \boldsymbol{P}_{v} \boldsymbol{x}\right. \\
& \left.\quad+l_{v}\left(\boldsymbol{x}^{\top} \boldsymbol{P}_{v} \boldsymbol{x}-1\right)\right] \\
& =-l_{v}+\boldsymbol{b}^{\top} \boldsymbol{P}_{v} \boldsymbol{x}_{v, \mathrm{opt}}
\end{aligned}
$$

mit

$$
\boldsymbol{x}_{v_{\mathrm{opt}}}=\left(\boldsymbol{A}^{\top} \boldsymbol{P}_{v}+\boldsymbol{P}_{v} \boldsymbol{A}-l_{v} \boldsymbol{P}_{v}\right)^{-1} \boldsymbol{P}_{v} \boldsymbol{b}
$$

wobei

$l_{v}=\max \left\{\lambda \in \mathbb{R}^{n} \mid \lambda \in \operatorname{Spec}\left(\mathcal{M}_{v}\right), \lambda<\boldsymbol{b}^{\top} \boldsymbol{P}_{v} \boldsymbol{x}_{v, \mathrm{opt}}\right\}$,

mit

$$
\mathcal{M}_{v}=\left[\begin{array}{cc}
\boldsymbol{P}_{v}^{-\frac{1}{2}} \boldsymbol{A}^{\top} \boldsymbol{P}_{v}^{\frac{1}{2}}+\boldsymbol{P}_{v}^{\frac{1}{2}} \boldsymbol{A} \boldsymbol{P}_{v}^{-\frac{1}{2}} & -\boldsymbol{I} \\
-\boldsymbol{P}_{v}^{\frac{1}{2}} \boldsymbol{b}^{\top} \boldsymbol{P}_{v}^{\frac{1}{2}} & \boldsymbol{P}_{v}^{-\frac{1}{2}} \boldsymbol{A}^{\top} \boldsymbol{P}_{v}^{\frac{1}{2}}+\boldsymbol{P}_{v}^{\frac{1}{2}} \boldsymbol{A} \boldsymbol{P}_{v}^{-\frac{1}{2}}
\end{array}\right]
$$

7 Da $W(\boldsymbol{x}, v)$ quadratisch ist, gilt für $\boldsymbol{x}^{\top} \boldsymbol{P}_{v} \boldsymbol{b} \leq 0, W(\boldsymbol{x}, v)=W(-\boldsymbol{x}, v)$. Folglich reicht an dieser Stelle aus, die Untersuchung der Konvergenzrate für $\boldsymbol{x}^{\top} \boldsymbol{P}_{v} \boldsymbol{b} \geq 0$ zu analysieren. 
ist. Gl. (34) und (35) folgen für ein festes $v$ aus den Optimalitätsbedingungen erster Ordnung

$$
\begin{aligned}
\left(\boldsymbol{A}^{\top} \boldsymbol{P}_{v}+\boldsymbol{P}_{v} \boldsymbol{A}-l_{v} \boldsymbol{P}_{v}\right) \boldsymbol{x} & =\boldsymbol{P}_{v} \boldsymbol{b}, \\
\boldsymbol{x}^{\top} \boldsymbol{P}_{v} \boldsymbol{x} & =1 .
\end{aligned}
$$

Da in Gl. (36) $\boldsymbol{P}_{v} \boldsymbol{b}=\left(\boldsymbol{b}^{\top} \boldsymbol{P}_{v}\right)^{\top}=\left[\begin{array}{ll}\mathbf{0}^{\top} & r v^{-2}\end{array}\right]^{\top}$ mit $r>0$ angenommen werden kann, folgt, dass $\boldsymbol{P}_{v} \boldsymbol{b} \neq \mathbf{0}$ ist, und, somit, dass der Lagrange-Operator $l_{v}$ aus Gl. (35) keine Lösung des allgemeinen Eigenwertproblems $\left(\boldsymbol{A}^{\top} \boldsymbol{P}_{v}+\boldsymbol{P}_{v} \boldsymbol{A}-\right.$ $\left.l_{v} \boldsymbol{P}_{v}\right) \boldsymbol{x}=\mathbf{0}$ sein kann, d.h., dass die Matrix $\boldsymbol{A}^{\top} \boldsymbol{P}_{v}+\boldsymbol{P}_{v} \boldsymbol{A}-$ $l_{v} \boldsymbol{P}_{v}$ nichtsingulär ist, und dass ihre Inverse in Gl. (34) existiert. Die Matrix $\mathcal{M}_{v}$ ergibt sich durch Einsetzen der Gl. (36) in Gl. (37), d.h. aus

$$
\left(\boldsymbol{b}^{\top} \boldsymbol{P}_{v} \boldsymbol{\Psi}_{v}^{-1}\right) \boldsymbol{P}_{v}\left(\boldsymbol{\Psi}_{v}^{-1} \boldsymbol{P}_{v} \boldsymbol{b}\right)=1,
$$

$\operatorname{mit} \boldsymbol{\Psi}_{v}=\boldsymbol{A}^{\top} \boldsymbol{P}_{v}+\boldsymbol{P}_{v} \boldsymbol{A}-l_{v} \boldsymbol{P}_{v}$. Gl. (38) stellt eine Gleichung in $l_{v}$ dar und ist equivalent $\mathrm{zu}^{8}{ }^{8}$

$$
\operatorname{det}\left[\begin{array}{cc}
1 & \boldsymbol{b}^{\top} \boldsymbol{P}_{v} \boldsymbol{\Psi}_{v}^{-1} \\
\boldsymbol{\Psi}_{v}^{-1} \boldsymbol{P}_{v} \boldsymbol{b} & \boldsymbol{P}_{v}^{-1}
\end{array}\right]=0 .
$$

Gl. (39) kann umgeformt werden zu

$$
\operatorname{det}\left(\left[\begin{array}{cc}
1 & \mathbf{0}_{1, n} \\
\mathbf{0}_{n, 1} & \boldsymbol{P}_{v}^{-1}
\end{array}\right]-\left[\begin{array}{cc}
0 & \boldsymbol{b}^{\top} \boldsymbol{P}_{v} \boldsymbol{\Psi}_{v}^{-1} \\
\boldsymbol{\Psi}_{v}^{-1} \boldsymbol{P}_{v} \boldsymbol{b} & \mathbf{0}_{n, n}
\end{array}\right]\right)=0
$$

und somit $\mathrm{zu}^{9}$

$$
\operatorname{det}\left(\left[\begin{array}{cc}
1 & \mathbf{0}_{1, n} \\
\mathbf{0}_{n, 1} & \boldsymbol{P}_{v}^{-1}
\end{array}\right]-\boldsymbol{T}\left[\begin{array}{ll}
\boldsymbol{\Psi}_{v}^{-1} & \mathbf{0}_{n, n} \\
\mathbf{0}_{n, n} & \boldsymbol{\Psi}_{v}^{-1}
\end{array}\right] \boldsymbol{S}\right)=0,
$$

mit $\boldsymbol{T} \in \mathbb{R}^{n+1 \times 2 n}, \boldsymbol{S} \in \mathbb{R}^{2 n \times n+1}$, sowie

$$
\boldsymbol{T}=\left[\begin{array}{cc}
\boldsymbol{b}^{\top} \boldsymbol{P}_{v} & \mathbf{0}_{1, n} \\
\mathbf{0}_{n, n} & \boldsymbol{I}_{n}
\end{array}\right], \boldsymbol{S}=\left[\begin{array}{cc}
\mathbf{0}_{n, 1} & \boldsymbol{I}_{n} \\
\boldsymbol{P}_{v} \boldsymbol{b} & \mathbf{0}_{n, n}
\end{array}\right] .
$$

Gl. (40) ist im Weiteren equivalent $\mathrm{zu}^{10}$

$$
\operatorname{det}\left(\left[\begin{array}{ll}
\boldsymbol{\Psi}_{v} & \mathbf{0}_{n, n} \\
\mathbf{0}_{n, n} & \boldsymbol{\Psi}_{v}
\end{array}\right]-\boldsymbol{S}\left[\begin{array}{cc}
1 & \mathbf{0}_{1, n} \\
\mathbf{0}_{n, 1} & \boldsymbol{P}_{v}
\end{array}\right] \boldsymbol{T}\right)=0,
$$

d.h.

$$
\operatorname{det}\left(\left[\begin{array}{cc}
\boldsymbol{\Psi}_{v} & \mathbf{0}_{n, n} \\
\mathbf{0}_{n, n} & \boldsymbol{\Psi}_{v}
\end{array}\right]-\left[\begin{array}{cc}
\mathbf{0}_{n, n} & \boldsymbol{P}_{v} \\
\boldsymbol{P}_{v} \boldsymbol{b} \boldsymbol{b}^{\top} \boldsymbol{P}_{v} & \mathbf{0}_{n, n}
\end{array}\right]\right)=0 .
$$

8 Dies folgt aus der Anwendung des Schur-Komplements, vgl. [4], Fakt 8.2.3.

9 Vgl. [10] für eine ähnliche Umformung.

$10 \mathrm{Vgl}$. [4], Fakten 2.8.3 und 2.8.4.
Folglich gilt

$$
\operatorname{det}\left(\left[\begin{array}{cc}
\boldsymbol{A}^{\top} \boldsymbol{P}_{v}+\boldsymbol{P}_{v} \boldsymbol{A}-l_{v} \boldsymbol{P}_{v} & \boldsymbol{P}_{v} \\
\boldsymbol{P}_{v} \boldsymbol{b} \boldsymbol{b}^{\top} \boldsymbol{P}_{v} & \boldsymbol{A}^{\top} \boldsymbol{P}_{v}+\boldsymbol{P}_{v} \boldsymbol{A}-l_{v} \boldsymbol{P}_{v}
\end{array}\right]\right)=0 .
$$

Schließlich ergibt sich durch links und rechts Multiplizieren mit der Matrix $\boldsymbol{P}_{v}^{\frac{1}{2}} \boldsymbol{I}_{2 n}$

$$
\operatorname{det}\left(\mathcal{M}_{v}-l_{v} \boldsymbol{I}_{2 n}\right)=0 .
$$

Dies bedeutet, dass der Lagrange-Operator $l_{v}$ ein Eigenwert der Matrix $\mathcal{M}_{v}$ ist.

Die Konvergenzrate aus Gl. (33) ist für jedes $v \in[\underline{v}, \bar{v}]$ die maximal mögliche Konvergenzrate, die bei gleichbleibender parameterabhängiger Matrix $\boldsymbol{P}_{v}$ mit einem beschränkten Regelgesetz erzielt werden kann. Dies gilt, weil für das System $\dot{\boldsymbol{x}}=\boldsymbol{A} \boldsymbol{x}-\boldsymbol{b} \operatorname{sgn}\left(\boldsymbol{b}^{\top} \boldsymbol{P}_{v} \boldsymbol{x}\right)$ für jedes $|u| \leq 1$ gilt

$$
\begin{aligned}
\dot{W}(\boldsymbol{x}, v) & =\boldsymbol{x}^{\top}\left(\boldsymbol{A}^{\top} \boldsymbol{P}_{v}+\boldsymbol{P}_{v} \boldsymbol{A}\right) \boldsymbol{x}-2 \boldsymbol{b}^{\top} \boldsymbol{P}_{v} \boldsymbol{x} \operatorname{sgn}\left(\boldsymbol{b}^{\top} \boldsymbol{P}_{v} \boldsymbol{x}\right) \\
& \leq \boldsymbol{x}^{\top}\left(\boldsymbol{A}^{\top} \boldsymbol{P}_{v}+\boldsymbol{P}_{v} \boldsymbol{A}\right) \boldsymbol{x}-2 \boldsymbol{b}^{\top} \boldsymbol{P}_{v} \boldsymbol{x} u .
\end{aligned}
$$

Entlang einer Trajektorie des Systems $\dot{\boldsymbol{x}}=\boldsymbol{A x}-$ $\boldsymbol{b} \operatorname{sgn}\left(\boldsymbol{b}^{\top} \boldsymbol{P}_{v} \boldsymbol{x}\right)$ ist der Abklingfaktor $-\dot{W}(\boldsymbol{x}, v)$ folglich immer größer als $2 \underline{\alpha}_{W}(v)$. Darüber hinaus ist dessen Variation durch den Abklingfaktor auf der Schaltfläche $s(\boldsymbol{x}(t), v(\boldsymbol{x}(t)))=\boldsymbol{b}^{\top} \boldsymbol{P}_{v} \boldsymbol{x}=0$ bestimmt, wenn die Trajektorie auf dieser Schaltfläche bleibt. Dies geschieht nach einer endlichen Zeit falls für $s \neq 0, s \dot{s}<0$ ist. Da

$$
\begin{aligned}
s \dot{s} & =s\left(\frac{\partial s}{\partial v} \frac{\partial v}{\partial \boldsymbol{x}^{\top}} \dot{\boldsymbol{x}}+\frac{\partial s}{\partial \boldsymbol{x}^{\top}} \dot{\boldsymbol{x}}\right) \\
& =s\left[\frac{\partial s}{\partial v}\left(-\frac{\partial g(\boldsymbol{x}, v) / \partial \boldsymbol{x}^{\top}}{\partial g(\boldsymbol{x}, v) / \partial v}\right)+\boldsymbol{b}^{\top} \boldsymbol{P}_{v}\right] \dot{\boldsymbol{x}} \\
& =s\left[\boldsymbol{b}^{\top} \boldsymbol{P}_{v}^{\prime} \boldsymbol{x}\left(-\frac{2 \boldsymbol{x}^{\top} \boldsymbol{P}_{v}}{\boldsymbol{x}^{\top} \boldsymbol{P}_{v}^{\prime} \boldsymbol{x}}\right)+\boldsymbol{b}^{\top} \boldsymbol{P}_{v}\right](\boldsymbol{A} \boldsymbol{x}-\boldsymbol{b} \operatorname{sgn}(s)) \\
& =s[\boldsymbol{b}^{\top} \boldsymbol{P}_{v}^{\prime} \boldsymbol{x} \underbrace{\left.\left(\frac{\boldsymbol{x}^{\top}\left(\boldsymbol{A}^{\top} \boldsymbol{P}_{v}+\boldsymbol{P}_{v} \boldsymbol{A}\right) \boldsymbol{x}}{\boldsymbol{x}^{\top}\left(-\boldsymbol{P}_{v}^{\prime}\right) \boldsymbol{x}}\right)+\boldsymbol{b}^{\top} \boldsymbol{P}_{v} \boldsymbol{A x}\right]} \underbrace{\left[\boldsymbol{b}^{\top} \boldsymbol{P}_{v}^{\prime} \boldsymbol{x}\left(-\frac{2 \boldsymbol{x}^{\top} \boldsymbol{P}_{v} \boldsymbol{b}}{\boldsymbol{x}^{\top} \boldsymbol{P}_{v}^{\prime} \boldsymbol{x}}\right)+\boldsymbol{b}^{\top} \boldsymbol{P}_{v} \boldsymbol{b}\right]}_{\delta(\boldsymbol{x}, v)}|s| \\
\varepsilon(\boldsymbol{x}, v) & \left(\left|\boldsymbol{b}^{\top} \boldsymbol{P}_{v}^{\prime} \boldsymbol{x}\right||\delta(\boldsymbol{x}, v)|+\left|\boldsymbol{b}^{\top} \boldsymbol{P}_{v} \boldsymbol{A} \boldsymbol{x}\right|-\varepsilon(\boldsymbol{x}, v)\right)|s| \\
& \leq\left(\left|\boldsymbol{b}^{\top}\left(\boldsymbol{P}_{v}^{\prime} \Delta_{v}\right) \boldsymbol{x}\right|+\left|\boldsymbol{b}^{\top} \boldsymbol{P}_{v} \boldsymbol{A} \boldsymbol{x}\right|-\varepsilon(\boldsymbol{x}, v)\right)|s|,
\end{aligned}
$$

mit $\Delta_{v}=\max _{x \in \partial \mathcal{E}\left(P_{v}, 1\right)}|\delta(\boldsymbol{x}, v)|$ und

$$
\Delta_{v}=\max \left\{\frac{\left|\lambda_{\min }\left(\boldsymbol{A}^{\top} \boldsymbol{P}_{v}+\boldsymbol{P}_{v} \boldsymbol{A}\right)\right|}{\lambda_{\max }\left(-\boldsymbol{P}_{v}^{\prime}\right)}, \frac{\left|\lambda_{\max }\left(\boldsymbol{A}^{\top} \boldsymbol{P}_{v}+\boldsymbol{P}_{v} \boldsymbol{A}\right)\right|}{\lambda_{\text {min }}\left(-\boldsymbol{P}_{v}^{\prime}\right)}\right\}
$$

gilt, ist es eine hinreichende Bedingung für das Auftreten eines Gleitzustandes um $s=0$, dass die Menge $S_{v}=\{\boldsymbol{x} \in$ 
$\left.\mathbb{R}^{n}|| \boldsymbol{b}^{\top}\left(\boldsymbol{P}_{v}^{\prime} \Delta_{v}\right) \boldsymbol{x}|+| \boldsymbol{b}^{\top} \boldsymbol{P}_{v} \boldsymbol{A x} \mid-\mathcal{\varepsilon}(\boldsymbol{x}, v)<0\right\}$ nichtleer ist. Dabei gilt noch

$$
\begin{aligned}
& \mathcal{E}(\boldsymbol{x}, v)=\frac{\partial s^{2} / \partial v}{\boldsymbol{x}^{\top}\left(-\boldsymbol{P}_{v}^{\prime}\right) \boldsymbol{x}}+\boldsymbol{b}^{\top} \boldsymbol{P}_{v} \boldsymbol{b} \\
& =\frac{\boldsymbol{x}^{\top}\left(\boldsymbol{P}_{v}^{\prime} \boldsymbol{b} \boldsymbol{b}^{\top} \boldsymbol{P}_{v}+\boldsymbol{P}_{v} \boldsymbol{b} \boldsymbol{b}^{\top} \boldsymbol{P}_{v}^{\prime}\right) \boldsymbol{x}}{\boldsymbol{x}^{\top}\left(-\boldsymbol{P}_{v}^{\prime}\right) \boldsymbol{x}}+\boldsymbol{b}^{\top} \boldsymbol{P}_{v} \boldsymbol{b} \\
& \geq \min _{x \in \partial \mathcal{E}\left(\boldsymbol{P}_{v}, 1\right)} \frac{\boldsymbol{x}^{\top}\left(\boldsymbol{P}_{v}^{\prime} \boldsymbol{b} \boldsymbol{b}^{\top} \boldsymbol{P}_{v}+\boldsymbol{P}_{v} \boldsymbol{b} \boldsymbol{b}^{\top} \boldsymbol{P}_{v}^{\prime}\right) \boldsymbol{x}}{\boldsymbol{x}^{\top}\left(-\boldsymbol{P}_{v}^{\prime}\right) \boldsymbol{x}}+\boldsymbol{b}^{\top} \boldsymbol{P}_{v} \boldsymbol{b} \\
& =\lambda_{\max }\left[\left(\boldsymbol{P}_{v}^{\prime} \boldsymbol{b} \boldsymbol{b}^{\top} \boldsymbol{P}_{v}+\boldsymbol{P}_{v} \boldsymbol{b} \boldsymbol{b}^{\top} \boldsymbol{P}_{v}^{\prime}\right)\left(-\boldsymbol{P}_{v}^{\prime^{-1}}\right)\right]+\boldsymbol{b}^{\top} \boldsymbol{P}_{v} \boldsymbol{b} \\
& =v^{-2}\left(\lambda_{\max }\left[\left(\boldsymbol{P}_{1}^{\prime} \boldsymbol{b} \boldsymbol{b}^{\top} \boldsymbol{P}_{1}+\boldsymbol{P}_{1} \boldsymbol{b} \boldsymbol{b}^{\top} \boldsymbol{P}_{1}^{\prime}\right)\left(-\boldsymbol{P}_{1}^{\prime^{-1}}\right)\right]+\boldsymbol{b}^{\top} \boldsymbol{P}_{1} \boldsymbol{b}\right),
\end{aligned}
$$

wobei $^{11} \lambda_{\max }\left[\left(\boldsymbol{P}_{1}^{\prime} \boldsymbol{b} \boldsymbol{b}^{\top} \boldsymbol{P}_{1}+\boldsymbol{P}_{1} \boldsymbol{b} \boldsymbol{b}^{\top} \boldsymbol{P}_{1}^{\prime}\right)\left(-\boldsymbol{P}_{1}^{\prime^{-1}}\right)\right] \in \mathbb{R}$ und, weil die Matrix $\boldsymbol{P}_{1}$ positiv definit ist, $\boldsymbol{b}^{\top} \boldsymbol{P}_{1} \boldsymbol{b}>0$. Folglich ist für

$$
\lambda_{\max }\left[\left(\boldsymbol{P}_{1}^{\prime} \boldsymbol{b} \boldsymbol{b}^{\top} \boldsymbol{P}_{1}+\boldsymbol{P}_{1} \boldsymbol{b} \boldsymbol{b}^{\top} \boldsymbol{P}_{1}^{\prime}\right)\left(-\boldsymbol{P}_{1}^{\prime^{-1}}\right)\right] \geq 0
$$

die Menge $S_{v}$ nichtleer. Daraus folgt, dass die Trajektorien des Systems, welche in der Menge $S_{v}$ starten, die Schaltfläche $s=0$ in endlicher Zeit erreichen und diese nicht mehr verlassen. Der Abklingfaktor $-\dot{W}(\boldsymbol{x}, v)$ ist aber innerhalb des Unterraums $s=0$ durch die Wahl der Matrix $\boldsymbol{P}_{v}$ beeinflussbar. Dies ergibt sich aus

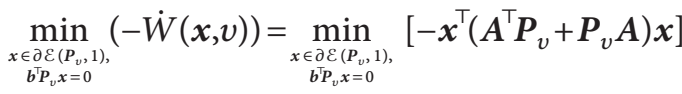

$$
\begin{aligned}
& =\min _{\substack{\left.x \in \partial \mathcal{x} \boldsymbol{P}_{v}, 1\right), b^{\top} \boldsymbol{P}_{v}=0}}\left[-\boldsymbol{x}^{\top}\left(\left(\boldsymbol{A}-\boldsymbol{b} \boldsymbol{k}_{v}^{\top}\right)^{\top} \boldsymbol{P}_{v}+\boldsymbol{P}_{v}\left(\boldsymbol{A}-\boldsymbol{b} \boldsymbol{k}_{v}^{\top}\right)\right) \boldsymbol{x}\right] \\
& \geq \min _{x \in \partial \mathcal{Q}\left(p_{v}, 1\right)}\left[-\boldsymbol{x}^{\top}\left(\left(\boldsymbol{A}-\boldsymbol{b} \boldsymbol{k}_{v}^{\top}\right)^{\top} \boldsymbol{P}_{v}+\boldsymbol{P}_{v}\left(\boldsymbol{A}-\boldsymbol{b} \boldsymbol{k}_{v}^{\top}\right)\right) \boldsymbol{x}\right] \\
& =-\frac{1}{v} \lambda_{\max }\left[\left(\hat{A}_{1}^{\top} \boldsymbol{P}_{1}+\boldsymbol{P}_{1} \hat{A}_{1}\right) \boldsymbol{P}_{1}^{-1}\right],
\end{aligned}
$$

mit $\hat{\boldsymbol{A}}_{1}=\boldsymbol{A}-\boldsymbol{b} \boldsymbol{k}_{1}^{\top}$, siehe Gl. (32). Die Konstante aus Gl. (41) kann man dabei durch die Wahl eines Regelgesetzes $\boldsymbol{u}=-\boldsymbol{k}_{1}^{\top} \boldsymbol{x}$ beeinflussen, wie in Bemerkung 3 erläutert. Die Konstante entspricht dem größten Realteil der Eigenwerte der Matrix $\hat{A}_{1}$ wenn man die Abklingrate der Funktion $W(\boldsymbol{x}, 1)$ entlang der Trajektorien der mit $u=-\boldsymbol{k}_{1}^{\top} \boldsymbol{x}$ geregelten Strecke maximiert. Je höher die Abklingrate ist, d.h. je stärker der Regler $\boldsymbol{u}=-\boldsymbol{k}_{1}^{\top} \boldsymbol{x}$ ist, desto größer wird der Abklingfaktor $-\dot{W}(\boldsymbol{x}, v)$ in dem Unterraum $s=0$ sein. Dabei wird jedoch das maximale Einzugsgebiet der Ruhleage kleiner, wie das Beispiel aus dem nächsten Abschnitt zeigt.

11 Vgl. [17], Fakt 8.15.21 und Fakt 6.52.

\subsection{Beispiel}

Als Beispiel wird die instabile Strecke aus [12],

$$
\dot{\boldsymbol{x}}=\left[\begin{array}{rr}
0.6 & -0.8 \\
0.8 & 0.6
\end{array}\right]+\left[\begin{array}{l}
2 \\
4
\end{array}\right] u,|u| \leq 1,
$$

betrachtet. Das Anfangsgebiet ist vorerst auf $X_{0}=\{\boldsymbol{x} \in$ $\left.\mathbb{R}^{2} \mid \boldsymbol{x}^{\top} \boldsymbol{x}=1\right\}$ festgelegt. Ziel eines ersten Entwurfes (Entwurf 1) ist die Maximierung der Konvergenzrate der quadratischen Funktion $W(\boldsymbol{x}, 1)=\boldsymbol{x}^{\top} \boldsymbol{P}_{1} \boldsymbol{x}$ innerhalb des kontraktiv invarianten Gebietes $\mathcal{E}\left(\boldsymbol{P}_{1}, 1\right)$, d.h.

$$
\begin{aligned}
& \max _{\boldsymbol{P}_{1}, \boldsymbol{h}_{1}, \boldsymbol{k}_{1}} \alpha, \text { sodass } \\
& \dot{W}(\boldsymbol{x}, 1) \leq-\alpha W(\boldsymbol{x}, 1), \\
& x_{0} \subset \mathcal{E}\left(\boldsymbol{P}_{1}, 1\right), \\
& \mathcal{E}\left(\boldsymbol{P}_{v}, 1\right) \subset\left\{\boldsymbol{x} \in \mathbb{R}^{n}|| \boldsymbol{h}_{v}^{\top} \boldsymbol{x} \mid \leq 1\right\}, \\
& \mathcal{E}\left(\boldsymbol{P}_{v}, 1\right) \subset\left\{\boldsymbol{x} \in \mathbb{R}^{n}|| \boldsymbol{k}_{v}^{\top} \boldsymbol{x} \mid \leq \beta\right\}, \\
& \mathcal{E}\left(\boldsymbol{P}_{v}, 1\right) \text { sind für } u_{\mathrm{h}}=-\boldsymbol{h}_{v}^{\top} \boldsymbol{x} \text { und } u_{\mathrm{k}}=-\boldsymbol{k}_{v}^{\top} \boldsymbol{x}
\end{aligned}
$$

ineinander verschachtelt und kontraktiv invariant.

In Gl. (46) stellt $\beta$ eine virtuelle Stellgrößenbeschränkung für den theoretisch unbeschränkten Regler $\boldsymbol{u}=-\boldsymbol{k}_{v}^{\top} \boldsymbol{x}$ dar, die zu hohe Verstärkungen verhindert. Wie in [12] gezeigt, kann der Reglerentwurf als konvexes Optimierungsproblem formuliert und numerisch gelöst werden. Es ergeben sich folgende Reglerkoeffizienten: ${ }^{12}$

$$
\begin{aligned}
\underline{v} & =0.2, \beta=3, \alpha=1.3348, \\
\boldsymbol{h}_{1}^{\top} & =\left[\begin{array}{ll}
0.7536 & 1.9034
\end{array}\right], \boldsymbol{k}_{1}^{\top}=\left[\begin{array}{ll}
1.6320 & 3.7628
\end{array}\right], \\
\boldsymbol{P}_{1} & =\left[\begin{array}{ll}
6.9205 & 2.5482 \\
2.5482 & 3.8181
\end{array}\right] .
\end{aligned}
$$

Abbildung 2 zeigt verschiedene ineinander verschachtelte Ellipsen, sowie das Gebiet der Anfangsauslenkungen $X_{0}$, den Bereich $\mathcal{L}\left(\boldsymbol{k}_{1}^{\top}, \beta\right)$ und die asymptotisch nullsteuerbare Region ${ }^{13} \mathcal{C}^{a}$ des Systems. Aufgrund des Entwurfsziels, d.h. der Maximierung der Konvergenzrate der quadratischen Funktion $W(\boldsymbol{x}, v)$ entlang der Trajektorien der mit dem schwächsten Regler geregelten Strecke, d.h. für $v=1$, ist es ersichtlich, dass die größte Ellipse gerade so groß ist, dass das Gebiet der Anfangsauslenkungen davon eingeschlossen ist. Eine Vergrößerung der virtuel-

12 Der Entwurf erfolgt nach der Transformation in die Steuerungsnormalform, die Ergebnisse sind in Steuerungsnormalform dargestellt.

13 Gemeint ist die Menge der Zustandspunkte, die mit einem beschränkten Regelgesetz erreichbar sind, vgl. [8]. 


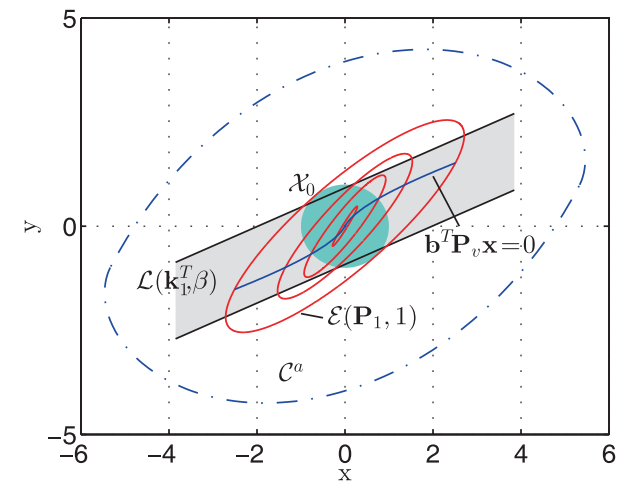

Abbildung 2: Ljapunov-Gebiete $\mathcal{E}\left(\boldsymbol{P}_{v}, 1\right)$ für verschiedene Werte von $v \in(0,1]$ und asymptotisch null-steuerbare Region $\mathrm{C}^{a}$.

len Stellgrößenbeschränkung $\beta$ würde die gleiche Ellipse erzeugen. Diese Gebiete sind unabhängig von dem untersuchten Regelgesetz, sodass man anhand des Satzes 2 das konvergenzoptimale Regelgesetz $u=-\operatorname{sgn}\left(\boldsymbol{b}^{\top} \boldsymbol{P}_{v} \boldsymbol{x}\right)$ verwenden kann.

Abbildung 3 zeigt die Variation von $\dot{W}(\boldsymbol{x})$ auf der äußersten Ellipse ( $v=1$ ) für das konvergenzoptimale Regelgesetz $u_{\text {sgn }}=-\operatorname{sgn}\left(\boldsymbol{b}^{\top} \boldsymbol{P}_{v} \boldsymbol{x}\right)$, sowie für das sättigende Regelgesetz $u_{\mathrm{k}}=-\operatorname{sat}\left(\boldsymbol{k}_{v}^{\top} \boldsymbol{x}\right)$ und das nichtsättigende Regelgesetz $u_{\mathrm{h}}=-\boldsymbol{h}_{v}^{\top} \boldsymbol{x}$ aus [12]. Dabei ist $\theta$ der Winkel des Zustandsvektors auf der Ellipse. Es ist ersichtlich, dass das hier vorgestellte Regelgesetz den kleinsten Wert von $\dot{W}(\boldsymbol{x})$ für jedes $\boldsymbol{x} \in \partial \mathcal{E}\left(\boldsymbol{P}_{1}, 1\right)$ erzielt. Auch mit sinkendem $v$, d.h. auf kleineren Ellipsen, erzielt das hier vorgestellte Regelgesetz den kleinsten Wert von $\dot{W}(\boldsymbol{x})$ für jedes $\boldsymbol{x} \in$ $\partial \mathcal{E}\left(\boldsymbol{P}_{v}, 1\right)$. Das bedeutet, dass für jedes $\boldsymbol{x} \in \partial \mathcal{E}\left(\boldsymbol{P}_{v}, 1\right)$ das hier vorgestellte Regelgesetz prinzipiell den schnellsten Abstieg von $v$ ermöglicht. Dabei gilt: je schneller $v$ sinkt, desto schneller werden die inneren Ellipsen erreicht, d.h. desto schneller wird der Ausregelvorgang sein.

Ein Vergleich der durchschnittlichen Ausregelzeiten für 360 äquidistante Anfangsauslenkungen auf der äußersten Ellipse wird in Tabelle 1 gezeigt. Die Ausregelzeit bezeichnet dabei die zeitliche Grenze, wann die Zustandsnorm $\|\boldsymbol{x}\|$ den Wert 0.1 zum letzten Mal unterschreitet. Verglichen wurden dabei das nichtsättigende Regelgesetz $u_{\mathrm{h}}=-\boldsymbol{h}_{v}^{\top} \boldsymbol{x}$, das sättigende Regelgesetz $\boldsymbol{u}_{\mathrm{k}}=-\operatorname{sat}\left(\boldsymbol{k}_{v}^{\top} \boldsymbol{x}\right)$, das hier vorgestellte Regelgesetz $u_{\mathrm{sgn}}=-\operatorname{sgn}\left(\boldsymbol{b}^{\top} \boldsymbol{P}_{v} \boldsymbol{x}\right)$, sowie das Regelgesetz $u_{\mathrm{hl}}=-\operatorname{sgn}\left(\boldsymbol{b}^{\top} \boldsymbol{P}_{\gamma} \boldsymbol{x}\right)$ aus [9]. Die beiden letzten Regelgesetze unterscheiden sich nur durch die Selektionsstrategie, bzw. durch die Wahl der parameterabhängigen Matrix $\boldsymbol{P}_{v}$, wobei die äußersten und innersten Ellipsen gleich gewählt sind und entsprechend die gleiche Konvergenzrate aufweisen.

Es ist ersichtlich, dass bei diesem Entwurf das hier vorgestellte Regelgesetz zwar eine kürzere Ausregelzeit als

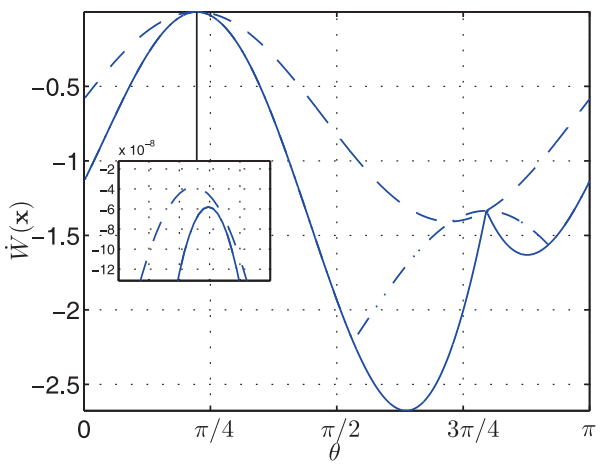

Abbildung 3: Variation von $\dot{W}(\boldsymbol{x})$ auf dem äußersten Ellipsoid, d.h. für $v=1$, bei verschiedenen Regelgesetzen: $u=-\operatorname{sgn}\left(\boldsymbol{b}^{\top} \boldsymbol{P}_{v} \boldsymbol{x}\right)(-)$, $u=-\operatorname{sat}\left(\boldsymbol{k}_{v}^{\top} \boldsymbol{x}\right)(-),. u=-\boldsymbol{h}_{v}^{\top} \boldsymbol{x}(--)$.

Tabelle 1: Durschnittliche Ausregelzeiten für 360 äquidistante Anfangsauslenkungen auf dem äußersten Ellipsoid. Die Ausregelzeit bezeichnet dabei die zeitliche Grenze, wann die Zustandsnorm $\|x\|$ den Wert 0.1 zum letzten Mal unterschreitet.

\begin{tabular}{lrrrr}
\hline$\varnothing \boldsymbol{t}_{\boldsymbol{a}}$ & $\boldsymbol{u}_{\mathrm{h}}$ & $\boldsymbol{u}_{\mathrm{k}}$ & $\boldsymbol{u}_{\mathrm{sgn}}$ & $\boldsymbol{u}_{\mathrm{hl}}$ \\
\hline Entwurf 1 & $5.6240 \mathrm{~s}$ & $3.0347 \mathrm{~s}$ & $3.8482 \mathrm{~s}$ & $6.5337 \mathrm{~s}$ \\
Entwurf 2 & $1.0901 \mathrm{~s}$ & $0.7804 \mathrm{~s}$ & $0.7780 \mathrm{~s}$ & $1.5675 \mathrm{~s}$ \\
\hline
\end{tabular}

das nichtsättigende Regelgesetz $u_{\mathrm{h}}=-\boldsymbol{h}_{v}^{\top} \boldsymbol{x}$ und als das Regelgesetz $u_{\mathrm{hl}}=-\operatorname{sgn}\left(\boldsymbol{b}^{\top} \boldsymbol{P}_{\gamma} \boldsymbol{x}\right)$ aus [9], jedoch eine längere Ausregelzeit als das Regelgesetz $u_{\mathrm{k}}=-\operatorname{sat}\left(\boldsymbol{k}_{v}^{\top} \boldsymbol{x}\right)$ aus [12] aufweist und dies obwohl das hier vorgestelte Regelgesetz so entworfen ist, dass der schnellste Abstieg von $v$ ermöglicht werden soll. Der Grund dafür liegt in dem Auftreten eines Gleitzustands um $\boldsymbol{s}=\boldsymbol{b}^{\top} \boldsymbol{P}_{v} \boldsymbol{x}=0$ (für $\boldsymbol{x} \in \mathbb{R}^{2}$ eine Kurve, vgl. Abbildung 2) während des Ausregelvorgangs.

In einem alternativen Entwurf (Entwurf 2) wird eine gewünschte Konvergenzrate $\alpha$ festgelegt und das kontraktiv invariante Gebiet $\mathcal{E}\left(\boldsymbol{P}_{1}, 1\right)$ maximiert ${ }^{14}$, d.h.

$$
\begin{aligned}
& \max _{\boldsymbol{P}_{1}, \boldsymbol{h}_{1}, \boldsymbol{k}_{1}} \delta \text {, sodass } \\
& \quad \delta X_{0} \subset \mathcal{E}\left(\boldsymbol{P}_{1}, 1\right), \\
& \text { (43), (45), (46), (47). }
\end{aligned}
$$

Es ergeben sich folgende Reglerkoeffizienten:

$$
\begin{aligned}
\underline{v} & =0.2, \beta=3, \alpha=5.5, \delta=0.1764, \\
\boldsymbol{h}_{1}^{\top} & =\left[\begin{array}{ll}
8.9837 & 4.0722
\end{array}\right], \boldsymbol{k}_{1}^{\top}=\left[\begin{array}{ll}
18.9648 & 8.7895
\end{array}\right], \\
\boldsymbol{P}_{1} & =\left[\begin{array}{cc}
246.2669 & 47.6303 \\
47.6303 & 17.3201
\end{array}\right] .
\end{aligned}
$$

14 Vgl. [8] für ein ähnliches Entwurfsproblem. 


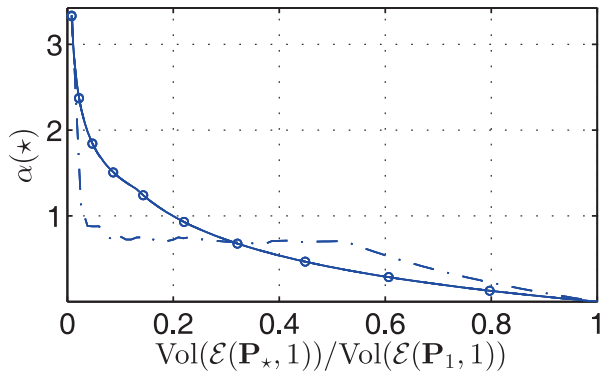

Abbildung 4: Variation von $\underline{\alpha}_{W}(\star)(\star=v, \gamma)$ (Entwurf 1) für die Regelgesetze: $u=-\operatorname{sgn}\left(\boldsymbol{b}^{\top} \boldsymbol{P}_{v} \boldsymbol{x}\right)(-), u=-\operatorname{sat}\left(k_{v} \boldsymbol{b}^{\top} \boldsymbol{P}_{v} \boldsymbol{x}\right)\left(-0, k_{v}=5\right)$, $u=-\operatorname{sgn}\left(\boldsymbol{b}^{\top} \boldsymbol{P}_{\gamma} \boldsymbol{x}\right)(-$.$) .$

Die Ergebnisse des zweiten Entwurfs im Bezug auf die durchschnittliche Ausregelzeit sind ebenfalls in Tabelle 1 gezeigt. Es ist dabei ersichtlich, dass das hier vorgestellte Regelgesetz die kürzeste Ausregelzeit aufweist, jedoch auf Kosten eines kleineren Einzugsgebietes der Ruhelage $(\delta=0.2)$. Die Verbesserung gegenüber dem ersten Entwurf ist bedingt durch die höhere Konvergenzrate in dem Unterraum $\boldsymbol{b}^{\top} \boldsymbol{P}_{v} \boldsymbol{x}=0$, welche durch ein größeres $\alpha$ nach unten beschränkt ist.

Abbildung 4 zeigt schließlich die Variation von $\underline{\alpha}_{W}(\star)$, mit $\star=v, \gamma$ für das Regelgesetz $u_{\text {sgn }}=-\operatorname{sgn}\left(\boldsymbol{b}^{\top} \boldsymbol{P}_{v} \boldsymbol{x}\right)$ sowie für das kontinuierliche Regelgesetz $u_{\mathrm{kv}}=-\operatorname{sat}\left(k_{v} \boldsymbol{b}^{\top} \boldsymbol{P}_{v} \boldsymbol{x}\right)$, mit $k_{v}=2$ und für das Regelgesetz $u_{\mathrm{hl}}=-\operatorname{sgn}\left(\boldsymbol{b}^{\top} \boldsymbol{P}_{\gamma} \boldsymbol{x}\right)$ aus [9]. Dabei wird die Variation von $\underline{\alpha}_{W}(\star)$ in Abhängigkeit von der relativen Fläche der Ellipse bezüglich der äußersten Ellipse gezeigt. Eine Verbesserung bezüglich der Konvergenzrate ist nicht im ganzen Bereich ersichtlich, jedoch stellt diese Konvergenzrate auch nur eine untere Grenze der tatsächlichen Konvergenzrate des Gesamtsystems dar. Der Vergleich zwischen den durchschnittlichen Ausregelzeiten aus Tabelle 1 zeigt eine deutliche Verbesserung der Performance im Falle des hier vorgestellten Regelgesetzes. Dabei stellt sich die Frage ob dieses Ergebnis abhängig von der gewählten Strecke ist und wie man die Variation der parameterabhängigen Matrix $\boldsymbol{P}_{v}$ wählen kann, sodass die Ausregelvorgänge weiter verbessert werden. Die Beantwortung dieser Fragen bleibt Gegenstand weiterer Untersuchungen.

\section{Zusammenfassung}

In dieser Arbeit wurden nichtlineare Regelgesetze für lineare Systeme mit Stellgrößenbeschränkung untersucht. Ziel der Arbeit war es, das konvergenzoptimale Regelgesetz im Fall weich strukturvariabler Regelungen mittels impliziten Ljapunov-Funktionen herzuleiten und zu analysieren. Die Ergebnisse zeigen, dass, für diese Klasse, die optimale Variation der Konvergenzrate allein durch die Selektionsstrategie bestimmt wird. Das Regelgesetz, das die Konvergenzrate in jedem durch die Selektionsstrategie vorgegebenen Teil des Einzugsgebietes maximiert, lautet $u=-\operatorname{sgn}\left(\boldsymbol{b}^{\top} \boldsymbol{P}_{v} \boldsymbol{x}\right)$, wobei $\boldsymbol{P}_{v}$ eine parameterabhängige Matrix ist, welche das Einzugsgebiet der Ruhelage in infinitesimal dicht ineinander verschachtelte Ellipsoiden mit der Gleichung $\boldsymbol{x}^{\top} \boldsymbol{P}_{v} \boldsymbol{x}=1$ unterteilt. Diese Einteilung hängt zum einen von dem erzielten maximalen Einzugsgebiet der Ruhelage und zum anderen von der Variation von $\boldsymbol{P}_{v}$ ab.

Das hier vorgestellte Regelgesetz hat den Vorteil, dass dessen Entwurf an bereits mit Hilfe von LMIs formulierten konvexen Optimierungsproblemen anknüpfen kann und die errechneten numerischen Lösungen, d.h. die beschränkten Regelgesetze, welche unter Umständen konservativ sind, verbessern kann. Des Weiteren stellt die Einfachheit dieses Regelgesetzes einen weiteren Vorteil dar. Auch die hier analysierte untere Grenze der Konvergenzrate des nichtlinearen Gesamtsystems stellt einen vorteilhaften Indikator zum Performancevergleich zwischen verschiedenen Regelgesetzen innerhalb des gesamten Einzugsgebietes der Ruhelage dar.

Auch wenn die vorgestellte konvergenzoptimale Regelung im Allgemeinen zu einer schnellen Ausregelung führt, wie in den Beispielen aus Abschnitt 4.3 gezeigt, ist diese nicht zeitoptimal. Die Verallgemeinerung der Ergebnisse bezüglich der erzielten Performance bei verschiedenen Regelstrecken bleibt Gegenstand weiterer Untersuchungen. Darüber hinaus könnte die Frage untersucht werden, unter welchen Bedingungen das konvergenzoptimale Regelgesetz eine endliche Konvergenzzeit aufweist.

\section{Literatur}

1. J. Adamy. Strukturvariable Regelungen mittels impliziter Lyapunov-Funktionen, Nr. 271. In Fortschr.-Ber. VDI Reihe 8, VDI Verlag, Düsseldorf, 1991.

2. J. Adamy. Implicit Lyapunov functions and isochrones of linear systems. IEEE Transactions on Automatic Control, 50(6):874879, 2005.

3. J. Adamy und A. Flemming. Soft variable-structure controls: a survey. Automatica, 40:1821-1844, 2004.

4. D.S. Bernstein. Matrix mathematics: theory, facts, and formulas with application to linear systems theory. Princeton University Press, New Jersey, 2005.

5. D.S. Bernstein und A.N. Michel. A chronological bibliography on saturating actuators. International Journal of Robust and Nonlinear Control, 5:375-381, 1995.

6. S. Boyd, L.E. Ghaoui, E. Feron, und V. Balakrishnan. Linear matrix inequalities in system and control theory, Vol 15 in SIAM studies in applied mathematics, 1994. 
7. M. Buhl, P. Joos, und B. Lohmann. Sättigende weiche strukturvariable Regelung. at-Automatisierungstechnik, 56(6):316323, 2008.

8. T. Hu und Z. Lin. Control systems with actor saturation. Analysis and design. Birkhäuser, Boston, 2001.

9. T. Hu und Z. Lin. On improving the performance with bounded continuous feedback laws. IEEE Transactions on Automatic Control, 47(9):1570-1575, 2002.

10. T. Hu, Z. Lin, und Y. Shamash. On maximizing the convergence rate for linear systems with input saturation. IEEE Transactions on Automatic Control, 48(7):1249-1253, 2003.

11. B. Jasniewicz. Über weiche strukturvariable Regelung mittels impliziter Lyapunov-Funktionen - von der impliziten zur expliziten Regelung, Nr. 1169. In Fortschr.-Ber. VDI Reihe 8, VDI Verlag, Düsseldorf, 2010.

12. B. Jasniewicz, J. Adamy, und D. Domont-Yankulova. Vereinfachte schnelle Regelung von linearen Systemen mit StellgröBenbegrenzungen. at-Automatisierungstechnik, 59(2):84-93, 2011.

13. H. Kiendl und G. Schneider. Synthese nichtlinearer Regler für die Regelstrecke const $/ s^{2}$ aufgrund ineinandergeschachtelter abgeschlossener Gebiete beschränkter Stellgröße. Regelungstechnik und Prozeß-Datenverarbeitung, 20:289-296, 1972.

14. H. Lens und J. Adamy. Schnelle Regelung von linearen Systemen mit Stellgrößenbeschränkungen. atAutomatisierungstechnik, 57:70-79, 2009.

15. H. Lens, J. Adamy, und D. Domont-Yankulova. A fast nonlinear control method for linear systems with input saturation. Automatica, 47:857-860, 2011.

16. A. Polyakov, D. Efimov, und W. Perruquetti. Finite-time stabilization using implicit Lyapunov function technique. IFAC Nolcos 2013, Toulouse, Frankreich, S. 140-145, 2013.

17. G. A. F. Seber. A matrix handbook for statisticians. John Wiley \& Sons, Hoboken, New Jersey, 2007.

18. J.-J. Slotine und W. Li. Applied nonlinear control. Prentice Hall, Englewood Cliffs, 1991.

19. S. Tarbouriech und M. Turner. Anti-windup design: an overview of some recent advances and open problems. IET Control Theory and Applications, 3(1):1-19, 2007.

20. D. Yankulova. Entwurf strukturvariabler Regelungen mittels linearer Matrixungleichungen, Nr. 1175. In Fortschr.-Ber. VDI Reihe 8, VDI Verlag, Düsseldorf, 2010.

\section{Autoreninformationen}

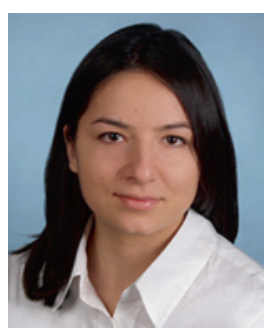

Dipl. Wirtsch.-Ing. Andreea Röthig Institut für Automatisierungstechnik und Mechatronik, Technische Universität Darmstadt, Landgraf-Georg-Str. 4, 64283 Darmstadt aroethig@rtr.tu-darmstadt.de

Dipl. Wirtsch.-Ing. Andreea Röthig promoviert am Fachgebiet Regelungsmethoden und Robotik der TU Darmstadt im Bereich nichtlineare Regelungen. Hauptarbeitsgebiete: Performance nichtlinearer
Regelungen, weiche strukturvariable Regelungen, Systeme mit Stellgrößenbeschränkung.

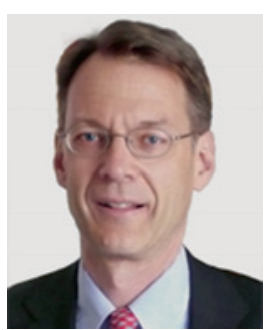

Prof. Dr.-Ing. Jürgen Adamy

Institut für Automatisierungstechnik und Mechatronik, Technische Universität

Darmstadt, Landgraf-Georg-Str. 4, 64283

Darmstadt

adamy@rtr.tu-darmstadt.de

Prof. Dr.-Ing. Jürgen Adamy ist Leiter des Fachgebietes Regelungsmethoden und Robotik im Fachbereich Elektrotechnik und Informationstechnik der TU Darmstadt. Hauptarbeitsgebiete: Regelungsverfahren, Computational Intelligence, autonome mobile Roboter.

Verfügbar unter

lediglich die vom Gesetz vorgesehenen Nutzungsrechte gemäß UrhG 